Algebraic 83 Geometric $\mathcal{T}_{\text {opology }}$

Volume 5 (2005) 835-864

Published: 24 July 2005

ATG

\title{
Counting immersed surfaces in hyperbolic 3-manifolds
}

\author{
Joseph D. MASTERS
}

\begin{abstract}
We count the number of conjugacy classes of maximal, genus $g$, surface subroups in hyperbolic 3-manifold groups. For any closed hyperbolic 3-manifold, we show that there is an upper bound on this number which grows factorially with $g$. We also give a class of closed hyperbolic 3 -manifolds for which there is a lower bound of the same type.
\end{abstract}

AMS Classification 57M50; 57N16, 57M27

Keywords Surface subgroups, bending, pleated surfaces, reflection orbifolds

\section{Introduction}

A major problem in the study of hyperbolic 3-manifolds is to determine the extent to which they contain useful surfaces. It is conjectured that every closed hyperbolic 3-manifold contains a number of immersed incompressible surfaces, each of which lifts to an embedding in a finite sheeted cover. The aim of this paper is to count the number in some appropriate sense.

We fix attention on a closed hyperbolic 3-manifold (or orbifold) $M$. Since we are interested in immersed surfaces up to homotopy, we shall be counting surface subgroups of $\pi_{1} M$ only up to conjugacy. Moreover, given our topological motivation, it is natural not to distinguish between a given immersed surface and one which covers it, and this leads to different ways of counting.

We define $s(M, g)$ to be the number of conjugacy classes of maximal surface groups of genus at most $g$ in $\pi_{1}(M)$. Two subgroups of $\pi_{1} M$ are said to be commensurable if their intersection has finite index in both. Let $s_{1}(M, g)$ denote the number of classes of surface groups in $\pi_{1} M$ under the equivalence relation generated by conjugacy and commensurability. Let $s_{2}(M, g)$ be the total number of conjugacy classes of surface subgroups in $\pi_{1} M$ of genus at most $g$. 
Thurston proved (Corollary 8.8.6 of [8]) that $s_{2}(M, g)$ is finite, but the proof does not give a practical upper bound. In [6], Soma gave an explicit upper bound, which is doubly exponential in the genus. We show:

Theorem 1.1 Let $M$ be a closed hyperbolic 3-manifold. Then there exists a constant $c_{2}>0$ such that, for large $g$,

$$
s_{2}(M, g)<e^{c_{2} g \log g} .
$$

We say that a totally geodesic immersion $f: S \rightarrow M$, is transverse if its image has a curve of transverse self-intersection; thus $f$ is not a finite cover of an embedding.

Theorem 1.2 Let $M$ be a closed hyperbolic 3-manifold, and suppose there is a transverse, totally geodesic immersion $f: S \rightarrow M$, for some hyperbolic surface $S$. Then there exist constants $c_{1}, c_{2}>0$ such that, for large $g$,

$$
e^{c_{1} g \log g}<s_{1}(M, g) \leq s(M, g) \leq s_{2}(g, M)<e^{c_{2} g \log g} .
$$

Theorem 1.2 may be viewed as a co-dimension one analogue of the Prime Geodesic Theorem for closed hyperbolic 3-manifolds (see 2]), which states that the number of conjugacy classes of maximal 1-manifold groups grows exponentially with the length of the corresponding geodesics. The 3-manifolds of Theorem 1.2 in this sense, grow faster in two dimensions than in one dimension. It is also interesting to count finite-index subgroups of $\pi_{1} M$ - see [4. For results on counting totally geodesic immersions of surfaces, see [5] and [3]. Anneke Bart and Brian Mangum have also obtained results on cutting and pasting immersed surfaces.

Organization In Section 2 we prove the upper bound. As in Thurston's finiteness proof, the first step is to homotop each immersed surface into pleated form. The rest of the proof relies on finding triangulations for the surfaces whose edges are sufficiently short, and counting the possible graphs which arise as 1-skeleta.

The proof of the lower bound is by an explicit construction of immersed incompressible surfaces, which is related to Thurston's bending deformation. We collect some preliminary material on surfaces and graphs in Section 3, and give the construction in Section 4. The main difficulty is to show that the resulting surface groups are inequivalent in the sense defined above. We do this by proving that they correspond to hyperbolic manifolds with non-isometric convex cores. 
Section 5 is devoted to examples where $M$ is a right-angled reflection orbifold. In this case, we are able to give more explicit, combinatorial proofs of both bounds. In particular, the proof of the upper bound avoids the usual theory of pleated surfaces, and as a corollary, we get an alternate proof of Thurston's finiteness theorem for these manifolds.

Theorem 1.3 Let $P \subset \mathbb{H}^{3}$ be a compact, right-angled polyhedron, and let $M$ be the associated reflection orbifold. Then for the constants of Theorem 1.2, we may take $c_{1}=1$ and $c_{2}=8 c(P)+1$, where $c(P)$ is the maximum number of edges in a face disk of $P$ (see Section 5 for definitions).

Thanks are due to Peter Shalen for a useful conversation, to Lewis Bowen for pointing out an error in a previous version, and to the University of Texas at Austin for its hospitality during the completion of this work.

\section{An upper bound}

In this section, we prove Theorem 1.1 .

If $G$ is a graph, let $\mathcal{V}(G)$ denote the vertex set of $G$, and let $\mathcal{E}(G)$ denote the edge set of $G$.

Lemma 2.1 There is a constant $k=k(s)$ such that any hyperbolic surface $S$ with $\operatorname{inj} \operatorname{rad}(S) \geq s$ contains an embedded graph $G$ satisfying:

(1) Every edge $e \in \mathcal{E}(G)$ is a geodesic arc of length $<s$,

(2) $|\mathcal{V}(G)|<k g$,

(3) degree $(v)<k$ for all vertices $v \in \mathcal{V}(G)$, and

(4) $S-G$ is a disjoint union of open disks.

Proof Let $\mathcal{B}=\left\{B_{1}, B_{2}, \ldots, B_{n}\right\}$ be a maximal collection of disjoint balls of radius $s / 4$ in $S$, and let $v_{i}$ denote the center of ball $B_{i}$. If $x, y \in S$ are points with $d(x, y)<s$, then there is a unique shortest arc in $S$ connecting them, which we denote $e(x, y)$.

Let

$$
\begin{aligned}
& \mathcal{S}=\left\{\left(v_{i}, v_{j}\right) \mid \text { int } B\left(v_{i}, s / 2\right) \cap \text { int } B\left(v_{j}, s / 2\right) \neq \emptyset\right\} \\
& \mathcal{E}=\left\{e\left(v_{i}, v_{j}\right) \mid\left(v_{i}, v_{j}\right) \in \mathcal{S}\right\}
\end{aligned}
$$


We define a subset $G$ of $S$ by:

$$
G=\bigcup_{e \in \mathcal{E}} e,
$$

and we give $G$ the structure of a graph by declaring the vertex set of $G$ to be:

$$
\mathcal{V}(G)=\bigcup_{e, e^{\prime} \in \mathcal{E}} e \cap e^{\prime},
$$

and then defining the edges of $G$ to be closures of components of $G-\mathcal{V}(G)$.

Lemma 2.2 There is a constant $k=k(s)$, (independent of $\mathcal{B}$ ) such that $|\mathcal{V}(G)|<k g$, and degree $(v)<k$ for all $v \in \mathcal{V}(G)$.

Proof We have:

$$
|\mathcal{B}| \leq \frac{\operatorname{Area}(S)}{\operatorname{Area}(B(x, s / 4))}=\frac{4 \pi(g-1)}{4 \pi \sinh ^{2}\left(\frac{s}{8}\right)},
$$

so $|\mathcal{B}|<m_{1} g$ for some $m_{1}$. If $B \subset S$ is a fixed $s / 4$-ball, there are at most a fixed number- call it $m_{2}$ - of disjoint $s / 4$-balls which will fit within an $s$ neighborhood of $B$. So we have

$$
|\mathcal{E}|=|\mathcal{S}|<m_{2}|\mathcal{B}|<m_{1} m_{2} g .
$$

Let $e \in \mathcal{E}$. Since length $(e)<s$, there are at most a fixed number- call it $m_{3}-$ of disjoint $s / 4$-balls in $S$ whose centers can fit within an $s$-neighborhood of $e$. Therefore, $e$ can intersect at most $\left(\begin{array}{c}m_{3} \\ 2\end{array}\right)$ different $e\left(v_{i}, v_{j}\right)$ 's. Since no pair of geodesic arcs of length less than $s$ can intersect more than once in $S$, we have

$$
\begin{aligned}
|\mathcal{V}(G)| & =\left|\bigcup_{e, e^{\prime} \in \mathcal{E}} e \cap e^{\prime}\right|, \\
& \leq\left(\begin{array}{c}
m_{3} \\
2
\end{array}\right)|\mathcal{E}| \\
& <m_{1} m_{2}\left(\begin{array}{c}
m_{3} \\
2
\end{array}\right) g .
\end{aligned}
$$

The fact that $e$ can intersect at most $\left(\begin{array}{c}m_{3} \\ 2\end{array}\right)$ other edges of $\mathcal{E}$ also implies

$$
\operatorname{deg}(v)<2\left(\begin{array}{c}
m_{3} \\
2
\end{array}\right)+2
$$

for all $v \in \mathcal{V}(G)$. Therefore we may take $k=\operatorname{Max}\left\{m_{1} m_{2}\left(\begin{array}{c}m_{3} \\ 2\end{array}\right), 2\left(\begin{array}{c}m_{3} \\ 2\end{array}\right)+2\right\}$.

Lemma 2.3 The surface $S-G$ is a disjoint union of open disks. 
Proof Let $\mathcal{C}=\left\{B\left(v_{1}, s / 2\right), \ldots, B\left(v_{n}, s / 2\right)\right\}$. Since $\mathcal{B}$ is maximal, $\mathcal{C}$ covers $S$ and therefore it is enough to show that every ball $B\left(v_{i}, s / 2\right) \in \mathcal{C}$ is covered by (the closures of) simply connected components of $S-G$.

The maximality of $\mathcal{C}$ implies that $\mathcal{C}-B\left(v_{i}, s / 2\right)$ covers $\partial B\left(v_{i}, s / 2\right)$. Let $B_{i 1}, \ldots, B_{i m} \in \mathcal{C}-B\left(v_{i}, s / 2\right)$ be balls which cover $\partial B\left(v_{i}, s / 2\right)$, arranged such that $B_{i j} \cap B_{i(j+1)} \neq \emptyset$ for all $j$. Then each triple $\left(v_{i}, v_{i j}, v_{i(j+1)}\right)$ is the vertex set of a geodesic triangle, $T_{j}$, contained in an $s$-ball (embedded, since $\operatorname{injrad}(S) \geq s$ ). Thus each $T_{j}$ bounds a disk $D_{j}$, which is the union of (closures of) components of $S-G$. The ball $B\left(v_{i}, s / 2\right)$ is covered by $\left\{D_{1}, \ldots, D_{m}\right\}$.

This concludes the proof of Lemma 2.1.

We now return to the proof of the upper bound. By [8], given any $\pi_{1}$-injective immersion $f: S \rightarrow M$, we can find a hyperbolic structure on $S$, and a homotopy of $f$ so that it is pleated with respect to this structure. Let $s=\operatorname{injrad}(M)$; since $f$ is pleated it takes closed loops in $S$ to closed loops of equal or shorter length in $M$, and therefore $\operatorname{inj} \operatorname{rad}(S) \geq s$. There is then a graph $G \subset S$, with the properties stated in Lemma 2.1.

Suppose $f^{\prime}: S^{\prime} \rightarrow M$ is another $\pi_{1}$-injective, pleated map of a genus $g$ surface, with a graph $G^{\prime} \subset S^{\prime}$ given by Lemma 2.1. With slight abuse of terminology, we say that $f$ and $f^{\prime}$ are homotopic if there is a map $g: S^{\prime} \rightarrow S$ such that $f g$ is homotopic to $f^{\prime}$.

Suppose there is a bijection $h: \mathcal{V}(G) \rightarrow \mathcal{V}\left(G^{\prime}\right)$, and label the vertices $\mathcal{V}(G)=$ $\left\{v_{1}, \ldots, v_{n}\right\}$, and $\mathcal{V}\left(G^{\prime}\right)=\left\{v_{1}^{\prime}, \ldots, v_{n}^{\prime}\right\}$, where $v_{i}^{\prime}=h v_{i}$. The map $h$ induces a graph $h(G)$ on $\mathcal{V}\left(G^{\prime}\right)$ by the rule that $v_{i}^{\prime}$ and $v_{j}^{\prime}$ are adjacent in $h(G)$ if and only if $v_{i}$ and $v_{j}$ are adjacent in $G$.

Lemma 2.4 Suppose that $d\left(f^{\prime} v_{i}^{\prime}, f v_{i}\right)<s / 4$ for all $i$, and that $h(G)=G^{\prime}$. Then $f$ and $f^{\prime}$ are homotopic.

Proof Let $\delta_{i}$ be a segment of length less than $s / 4$ connecting $f v_{i}$ and $f^{\prime} v_{i}^{\prime}$. Sliding the $v_{i}^{\prime}$ 's along the $\delta_{i}$ 's, we may homotope $\left.f^{\prime}\right|_{G^{\prime}}$ to a map $g: G^{\prime} \rightarrow M$, so that $g\left(v_{i}^{\prime}\right)=f\left(v_{i}\right)$ and so that $g G^{\prime}$ is contained in $f^{\prime} G^{\prime} \bigcup_{i} \delta_{i}$. If $E^{\prime}$ is an edge of $G^{\prime}$, then its image under $g$ is given by:

$$
g\left(E^{\prime}\right)=f^{\prime}\left(E^{\prime}\right) \cup \delta_{i} \cup \delta_{j}, \quad \text { (for some i,j), }
$$

which has total length less than $s$. Since $\operatorname{inj} \operatorname{rad}(M)=s$, any two segments of $M$ with the same endpoints which have length less than $s$ are homotopic, 
fixing endpoints. Therefore, the map $\left.f^{\prime}\right|_{G^{\prime}}$ is homotopic to $\left.f h\right|_{G^{\prime}}$. Since the complementary regions of $G$ and $G^{\prime}$ are disks, the map $h$ can be extended to a map $h: S^{\prime} \rightarrow S$, such that $f^{\prime}$ and $f h$ are homotopic.

Let $\mathcal{V}=\left\{v_{1}, \ldots, v_{k}\right\}$ be a fixed set of vertices, and let $\mathcal{G}(\mathcal{V})$ be the set of all graphs on $\mathcal{V}$. Note that the relation of equality between graphs on $\mathcal{V}$ is stronger than the relation of isomorphism between graphs on $\mathcal{V}$.

Let

$$
\mathcal{G}(\mathcal{V}, n)=\{G \in \mathcal{G}(\mathcal{V}) \mid \text { each vertex of } G \text { has degree at most } n\}
$$

A computation shows that

$$
|\mathcal{G}(\mathcal{V}, n)| \leq|\mathcal{V}|^{n|\mathcal{V}|}
$$

Let $k_{1}=k(s)$ be the constant provided by Lemma 2.1. Let $\mathcal{C}$ be a collection of balls of radius $s / 4$ which covers $M$, and let $k_{2} \geq|\mathcal{C}|$. Suppose we have a collection $\mathcal{S}$ of more than $\left(k_{1} g\right)\left(k_{1} g\right)^{k_{2}}\left(k_{1} g\right)^{k_{1}^{2} g}$ pleated maps $f_{i}: S_{i} \rightarrow M$, where $S_{i}$ is a hyperbolic surface of genus $g$. Associated to each $f_{i} \in \mathcal{S}$ is a graph $G_{i} \subset S_{i}$ satisfying the properties stated in Lemma 2.1. Since $\left|\mathcal{V}\left(G_{i}\right)\right|<k_{1} g$, there is a subset $\mathcal{S}_{1} \subset \mathcal{S}$ of $\left(k_{1} g\right)^{k_{2}}\left(k_{1} g\right)^{k_{1}^{2} g}$ pleated maps whose graphs all have the same number of vertices. Since there are at most $\left(k_{1} g\right)^{k_{2}}$ ways to map $\mathcal{V}\left(G_{i}\right)$ to $\mathcal{C}$, then there is a subset $\mathcal{S}_{2} \subset \mathcal{S}_{1}$ of $\left(k_{1} g\right)^{k_{1}^{2} g}$ pleated maps, such that, if we fix a map $f \in \mathcal{S}_{2}$ with associated graph $G$, then for any $f_{i} \in \mathcal{S}_{2}$, there are bijections $h_{i}: \mathcal{V}\left(G_{i}\right) \rightarrow \mathcal{V}(G)$, such that, for all $v \in \mathcal{V}\left(G_{i}\right), d\left(f h_{i}(v), f_{i}(v)\right) \leq$ $s / 4$. By Equation 1, there is a pair of distinct maps $f_{i}, f_{j} \in \mathcal{S}_{2}$ such that $h_{i}\left(G_{i}\right)=h_{j}\left(G_{j}\right)$. Therefore, by Lemma 2.4 $f_{i}$ is homotopic to $f_{j}$. This concludes the proof of the upper bound.

\section{Properties of graphs and surfaces}

By a metric graph, $G$, we mean a graph with a metric space structure, determined by the following procedure: we assign a length to each edge, $e$, and give $e$ a path metric induced from the interval $[0, \operatorname{length}(e)]$. The path metric on the edges then determines a path metric on $G$. The standard metric on $G$ is obtained by setting the length of each edge to be one. A map $f: X \rightarrow Y$ between metric spaces is a $(k, c)$-quasi-isometry $(k, c>1)$ if $\frac{d\left(f x_{1}, f x_{2}\right)}{k}-c<$ $d\left(x_{1}, x_{2}\right)<k d\left(f x_{1}, f x_{2}\right)+c$ for all $x_{1}, x_{2} \in X$, and $d(y, f(X))<c$ for all $y \in Y$. 
Lemma 3.1 Let $G$ and $G^{\prime}$ be metric graphs, with no vertices of degree 2, and with all edges longer than $u=6 k^{3}\left(k^{2}+3\right) c$. Then if $G$ and $G^{\prime}$ are $(k, c)$ quasi-isometric, they are isomorphic.

Proof Let $f: G \rightarrow G^{\prime}$ be a $(k, c)$-quasi-isometry. Then, there exist numbers $k^{\prime}, c^{\prime}$ and $s>0$, and a map $g: G^{\prime} \rightarrow G$, which is a $\left(k^{\prime}, c^{\prime}\right)$-quasi-isometry, such that $d(g f x, x)<s$, and $d(f g y, y)<s$ for all $x \in G$ and $y \in G^{\prime}$. In fact, we may take $k^{\prime}=k, c^{\prime}=3 k c$, and $s=k c$.

Let $x$ be a vertex of $G$ of degree $n>2$, and let $e_{1}, \ldots, e_{n}$ be distinct edges incident to $x$. Since $f$ is a $(k, c)$-quasi-isometry, any point of $f e_{i}$ is within distance $c$ of another point of $f e_{i}$, and $\operatorname{since} \operatorname{length}\left(e_{i}\right)>k\left(k^{2}+2\right) c$, then Diameter $\left(f e_{i}\right)>k\left(k^{2}+2\right) c / k-c=\left(k^{2}+1\right) c$. Thus, for each $i$, there are points $x_{i} \in e_{i}$ such that

$$
\left(k^{2}+1\right) c<d\left(f x_{i}, f x\right)<\left(k^{2}+1\right) c+c .
$$

Then

$$
\begin{aligned}
d\left(x_{i}, x\right) & >d\left(f x_{i}, f x\right) / k-\frac{c}{k} \\
& >\left(k^{2}+1\right) c / k-\frac{c}{k} \\
& =k c .
\end{aligned}
$$

Since edges of $G$ all have length greater than $2 d\left(x_{i}, x\right)$, then

$$
d\left(x_{i}, x_{j}\right)=d\left(x_{i}, x\right)+d\left(x_{j}, x\right)>2 k c,
$$

and so

$$
d\left(f x_{i}, f x_{j}\right)>(2 k c) / k-c>c .
$$

So, in the $\left(k^{2}+2\right) c$-neighborhood of $f x$, there are $n$ points $f x_{i}$, such that $\left|d\left(f x_{i}, f x\right)-d\left(f x_{j}, x\right)\right|<c$, and $d\left(f x_{i}, f x_{j}\right)>c$ for all $i \neq j$. This implies that $f x$ is within distance $\left(k^{2}+2\right) c$ of a vertex of $G^{\prime}$.

If $x$ is a degree 1 vertex, then we claim that $y=f x$ is within distance $k^{\prime}\left(k^{\prime 2}+\right.$ 2) $c^{\prime}$ of a degree 1 vertex of $G^{\prime}$. For suppose not. Then there is an interval $e$ of length $2 k^{\prime}\left(k^{\prime 2}+2\right) c^{\prime}$ isometrically embedded in $G^{\prime}$, such that $f x$ is the midpoint of $e$. Let $e_{1}$ and $e_{2}$ be sub-intervals of $e$ with $e_{1} \cap e_{2}=\{f x\}$, and length $\left(e_{i}\right)=$ $k^{\prime}\left(k^{\prime 2}+2\right) c^{\prime}$. Then Diameter $\left(g e_{i}\right)>\left[k^{\prime}\left(k^{\prime 2}+2\right) c^{\prime}\right] / k^{\prime}-c^{\prime}=\left(k^{\prime 2}+1\right) c^{\prime}$, so for both $i$ 's there exists a point $y_{i} \in e_{i}$ such that

$$
\left(k^{2}+1\right) c^{\prime}<d\left(g y_{i}, g y\right)<\left(k^{\prime 2}+1\right) c^{\prime}+c^{\prime} .
$$


We have:

$$
\begin{aligned}
d\left(y_{i}, y\right) & >\frac{d\left(g y_{i}, g y\right)}{k^{\prime}}-\frac{c^{\prime}}{k^{\prime}} \\
& >\frac{\left(k^{\prime 2}+1\right) c^{\prime}}{k^{\prime}}-\frac{c^{\prime}}{k^{\prime}} \\
& =k^{\prime} c^{\prime}
\end{aligned}
$$

Since all edges of $G^{\prime}$ have length greater than $d\left(y_{i}, y\right)$, then

$$
d\left(y_{1}, y_{2}\right)=d\left(y_{1}, y\right)+d\left(y_{2}, y\right)>2 k^{\prime} c^{\prime},
$$

and

$$
d\left(g y_{1}, g y_{2}\right)>\left(2 k^{\prime} c^{\prime}\right) / k^{\prime}-c^{\prime}=c^{\prime} .
$$

However, we have $d(g y, x)=d(g f x, x)<s=k c$, so $g y$ and $x$ are contained in the same edge. Since $x$ has degree one, and

$$
\begin{aligned}
d\left(g y_{i}, x\right) & >d\left(g y_{i}, g y\right)-d(g y, x) \\
& >\left(k^{\prime 2}+1\right) c^{\prime}-s \\
& =\left(k^{2}+1\right)(3 k c)-k c \\
& >k c=s \\
& >d(g y, x),
\end{aligned}
$$

then $d\left(g y_{i}, x\right)=d\left(g y_{i}, g y\right)+d(g y, x)$. Then

$$
\begin{aligned}
\left|d\left(g y_{1}, x\right)-d\left(g y_{2}, x\right)\right| & =\left|d\left(g y_{1}, g y\right)+d(g y, x)-\left(d\left(g y_{2}, g y\right)+d(g y, x)\right)\right| \\
& =\left|d\left(g y_{1}, g y\right)-d\left(g y_{2}, y\right)\right| \\
& <c^{\prime} .
\end{aligned}
$$

Since $d\left(g y_{i}, x\right)=d\left(g y_{i}, g y\right)+d(g y, x)<\left(k^{\prime 2}+2\right) c^{\prime}+s=\left(k^{2}+2\right)(3 k c)+k c$, then $g y_{i}$ and $x$ are contained in the same edge. Therefore $d\left(g y_{1}, g y_{2}\right)=\mid d\left(g y_{1}, x\right)-$ $d\left(g y_{2}, x\right) \mid<c^{\prime}$, for a contradiction. Therefore, $f x$ is within distance $k^{\prime}\left(k^{\prime 2}+2\right) c^{\prime}$ of a degree 1 vertex of $G^{\prime}$.

Let

$$
\begin{aligned}
t & =\operatorname{Max}\left(\left(k^{2}+2\right) c, k^{\prime}\left(k^{\prime 2}+2\right) c^{\prime}\right) \\
& =\operatorname{Max}\left(\left(k^{2}+2\right) c, k\left(k^{2}+2\right) 3 k c\right) \\
& =3 k^{2}\left(k^{2}+2\right) c \\
& <u / 2
\end{aligned}
$$

We have shown $f(\mathcal{V}(G)) \subset N_{t}\left(\mathcal{V}\left(G^{\prime}\right)\right)$. Since $t<u / 2$, there is an induced map $f^{*}: \mathcal{V}(G) \rightarrow \mathcal{V}\left(G^{\prime}\right)$, defined by $f^{*} v=N_{u / 2}(f v) \cap \mathcal{V}\left(G^{\prime}\right)$. Similarly, if 
$t^{\prime}=\operatorname{Max}\left(\left(k^{\prime 2}+2\right) c, k\left(k^{2}+2\right) c\right)$, then $t^{\prime}<u / 2, g\left(\mathcal{V}\left(G^{\prime}\right)\right) \subset N_{t^{\prime}}(\mathcal{V}(G))$, and there is an induced map $g^{*}: \mathcal{V}\left(G^{\prime}\right) \rightarrow \mathcal{V}(G)$.

We have:

$$
\begin{aligned}
d\left(g f^{*} v, v\right) & \leq d(v, g f v)+d\left(g f v, g f^{*} v\right) \\
& \leq s+k^{\prime} d\left(f v, f^{*} v\right)+c^{\prime} \\
& \leq s+k\left(3 k^{2}\left(k^{2}+2\right) c\right)+3 k c \\
& <u / 2
\end{aligned}
$$

Therefore $g^{*} f^{*} v=v$, and similarly $f^{*} g^{*} v=v$ for all $v \in \mathcal{V}\left(G^{\prime}\right)$. So the maps $f^{*}$ and $g^{*}$ are inverses to each other, and hence bijections.

Furthermore, if $v_{1}, v_{2}$ are adjacent vertices in $G$, with an edge $e$ connecting them, then $f(e)$ is a $(k, c)$-quasi-geodesic segment, intersecting $N_{t}\left(f^{*} v_{1} \cup f^{*} v_{2}\right)$, but disjoint from $N_{t}\left(\mathcal{V}\left(G^{\prime}\right)-\left\{f^{*} v_{1}, f^{*} v_{2}\right\}\right)$, and this implies that $f^{*} v_{1}$ and $f^{*} v_{2}$ are adjacent in $G^{\prime}$. A similar statement holds for $g^{*}$, and so the map $f^{*}$ induces an isomorphism from $G$ to $G^{\prime}$.

Let $G$ be a graph, with a non-separating basepoint $p$ contained in the interior of an edge. Let $N(p)$ be a regular open neighborhood of $p$ in $G$, and let $G / p=G-$ $N(p)$. Then $G / p$ has the structure of a graph, with $\mathcal{V}(G / p)=\mathcal{V}(G) \cup\left\{v_{1}, v_{2}\right\}$, where $v_{1}$ and $v_{2}$ are degree one vertices. Say that the pairs $(G, p)$ and $\left(G^{\prime}, p^{\prime}\right)$ are inequivalent if the universal cover of $G / p$ is not isomorphic to the universal cover of $G^{\prime} / p^{\prime}$.

Lemma 3.2 Let $G$ be a bouquet of two circles, with basepoint $p$ in the interior of an edge. Then there is a constant $c>0$ such that, for all $n$, the number of inequivalent covers $(\tilde{G}, \tilde{p})$ of $(G, p)$ of degree $n$ is at least $(c n)$ !.

Proof Let $a, b$ be the edges of $G$, and assume $p \in b$. Any graph $\tilde{G}$ whose vertices all have degree four is a cover of $G$, and we may specify the covering map by directing the edges of $\tilde{G}$, and labeling each of them either $a$ or $b$. Let $\sigma$ be an order two permutation on $\{1, \ldots, n\}$. We consider $4 n$-fold covers $\pi:(\tilde{G}, \tilde{p}) \rightarrow(G, p)$ constructed as follows (see Figure 1):

(1) Begin with $4 n$ vertices $v_{1}, \ldots, v_{4 n}$, and, for all $i$, connect $v_{i}$ and $v_{i+1}$ with an edge labeled $a$.

(2) If either
(a) $i$ is even
(b) $i \geq 2 n$, or 
(c) $i=2 j-1$, with $j \leq n$, and $\sigma j=j$,

then attach a 1-cycle labeled $b$ to $v_{i}$.

(3) If $\sigma(i)=j$, and $i \neq j$, then attach a 2-cycle with edges labeled $b$ to $v_{2 i-1}, v_{2 j-1}$.

(4) Choose a basepoint, $\tilde{p}$, in the 1-cycle which is adjacent to $v_{0}$ and labeled $b$.

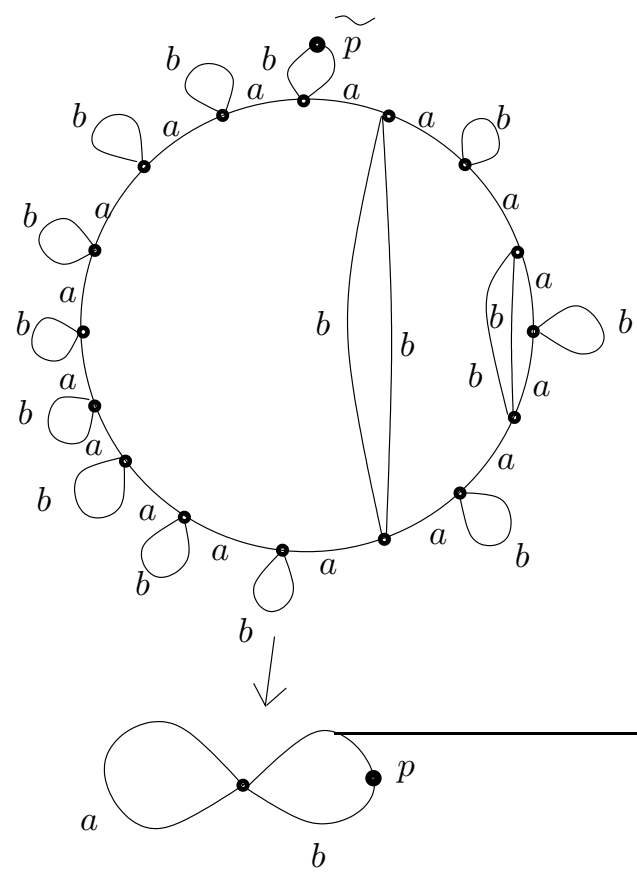

Figure 1: A cover of $G$, with $2 n=16$ and $\sigma=(1,2)(3,4)$

Let $\rho: T \rightarrow \tilde{G} / \tilde{p}$ be the universal cover. We give $T$ the standard path metric (see above). Let $\mathcal{E}_{1}$ be the set of edges of $T$ whose endpoints are equidistant from $\partial T$. Note that $\mathcal{E}_{1}$ is preserved under any isomorphism of $T$, and that $\mathcal{E}_{1}$ contains $\rho^{-1}$ of any 1 -cycle. Let $\mathcal{E}_{2}$ be the set of edges in $\mathcal{E}(G)-\mathcal{E}_{1}$ which are adjacent to at least two elements of $\mathcal{E}_{1}$. Then $\mathcal{E}_{2}$ is also isomorphism-invariant. Since no edge labeled $a$ has endpoints which are equidistant from $\partial(\tilde{G} / \tilde{p})$, then $\rho^{-1} \pi^{-1} a \cap \mathcal{E}_{1}=\emptyset$, and since every edge labeled $a$ in $\tilde{G}$ is adjacent to a 1 -cycle, then $\rho^{-1} \pi^{-1} a \subset \mathcal{E}_{2}$. Also, any element of $\rho^{-1} \pi^{-1} b-\mathcal{E}_{1}$ can be adjacent to (and distinct from) at most one element of $\mathcal{E}_{1}$, so $\rho^{-1} \pi^{-1} b \cap \mathcal{E}_{2}=\emptyset$. Therefore $\mathcal{E}_{2}=\rho^{-1} \pi^{-1} a$. 
Let $d_{a}: \mathcal{V}(T) \rightarrow \mathbb{Z}$ be the function defined by setting $d_{a}(x)$ to be the distance from $x$ to $\partial T$ along paths whose edges (except for the final edge) are all labeled $a$. Let $\mathcal{V}_{i}$ be the set of vertices of $T$ with $d_{a}(x)=i$. Since $\mathcal{E}_{2}=\rho^{-1} \pi^{-1} a$ is isomorphism-invariant, then $\mathcal{V}_{i}$ is isomorphism invariant. The transposition $(i j)$ occurs in the cycle decomposition of $\sigma$ if and only if there is an edge between $\mathcal{V}_{2 i}$ and $\mathcal{V}_{2 j}$, and thus the permutation $\sigma$ is determined by the isomorphism type of $T$. Since the number of such permutations grows as a factorial in $n$, the number of inequivalent covers $(\tilde{G}, \tilde{p})$ also grows as a factorial in $n$.

Lemma 3.3 Let $S$ be a hyperbolic surface, let $L$ be a non-separating collection of disjoint loops in $S$, and let $u$ be an arbitrary function of two variables. Then there is a finite cover $\tilde{S}$ of $S$, and a bouquet of two circles $G$ such that

(1) there is a $(k, c)$-quasi-isometry $g: \tilde{S} \rightarrow G$,

(2) both edges of $G$ have length greater than $u(k, c)$, and

(3) $g(\tilde{L})$ is a point, for some 1-1 lift $\tilde{L}$ of $L$.

Proof After passing to a preliminary cover, we may assume that $S-L$ has positive genus. Then there is a map of $S$ onto a bouquet of two circles, $g_{1}$ : $S \rightarrow G_{1}=a \cup b$, such that $g_{1}$ induces a surjection on fundamental groups, and $g_{1}(L)$ is a point. We give $G_{1}$ the standard metric (so $a$ and $b$ have length 1 ), and since $S$ and $G_{1}$ are compact, $g_{1}$ is a $\left(k, c_{1}\right)$-quasi-isometry for some $k, c_{1}$.

Let $c=c_{1}+2$, and let $s>u(k, c)$. Let $\pi: \tilde{G}_{1} \rightarrow G_{1}$ be a $2 s$-fold cover of $G_{1}$, where $\rho^{-1} a$ is a single cycle, and $\pi^{-1} b$ is a set of $2 s-2$ cycles, as indicated in Figure 2 There is an induced cover $\tilde{S}$ of $S$, to which $L$ lifts. Let $\tilde{b}$ be the component of $\pi^{-1} b$ which maps 2 to 1 onto $b$, and let $G$ be the graph $\pi^{-1} a$, with the vertices of $\tilde{b}$ identified to a point. Then $G$ is homeomorphic to a bouquet of two circles, and there is a $(1,2)$-quasi-isometry $g_{2}: \tilde{G}_{1} \rightarrow G$. Thus $g_{2} g_{1}: \tilde{S} \rightarrow G$ is a $(k, c)$-quasi-isometry, the edges of $G$ all have length at least $s>u(k, c)$, and there are 1-1 lifts $\tilde{L}$ of $L$ such that $g_{2} g_{1} \tilde{L}$ is a point.

Let $S$ be a closed hyperbolic surface, with a non-separating collection of loops $L$ in $S$, and let $(\tilde{S}, \tilde{L})$ be a finite cover of $S$, with a $1-1$ lift $\tilde{L}$ of $L$. Let $\tilde{S} / \tilde{L}$ be the metric completion of $\tilde{S}-\tilde{L}$, which is a hyperbolic surface with geodesic boundary, and let $P \subset \mathbb{H}^{2}$ be the universal cover of $\tilde{S} / \tilde{L}$. For another such cover $\left(\tilde{S}^{\prime}, \tilde{L}^{\prime}\right)$, we define analogous objects $\pi^{\prime}, \tilde{S}^{\prime}$ and $P^{\prime}$. We say that the pairs $(\tilde{S}, \tilde{L})$ and $\left(\tilde{S}^{\prime}, \tilde{L}^{\prime}\right)$ are inequivalent if $P$ is not isometric to $P^{\prime}$. 


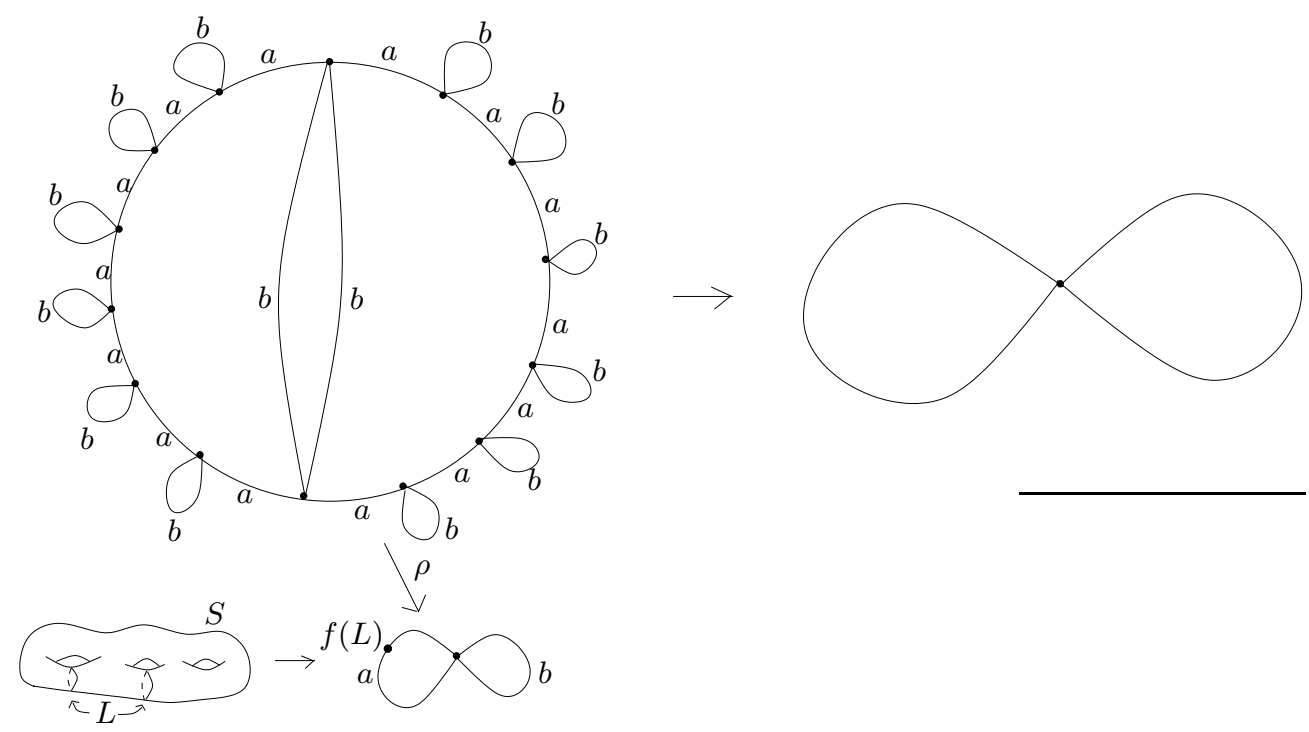

Figure 2: There is a cover of $\mathrm{S}$ which maps onto a bouquet of circles, with long edges.

Lemma 3.4 Let $(S, L)$ be as above. Then the number of inequivalent covers $(\tilde{S}, \tilde{L})$ of degree $n$ is at least $(c n)$ ! for some constant $c>0$.

Proof We replace $S$ with the cover given by Lemma 3.3. We are free to do this, since the effect on covering degrees is linear. We have a $(k, c)$-quasiisometry $g: S \rightarrow G$, where $G$ is a bouquet of two circles, whose lengths are at least $u(k, c)$, where $u$ is a function of $k$ and $c$ which we will determine later.

Let $(\tilde{G}, \tilde{p})$, and $\left(\tilde{G}^{\prime}, \tilde{p}^{\prime}\right)$ be inequivalent covers of $G$, with induced covers $(\tilde{S}, \tilde{L})$ and $\left(\tilde{S}^{\prime}, \tilde{L}^{\prime}\right)$ of $S$. Let $P$ (resp. $\left.P^{\prime}\right)$ be the universal cover of $\tilde{S} / \tilde{L}\left(\operatorname{resp} . \tilde{S}^{\prime} / \tilde{L}^{\prime}\right)$, and let $T$ (resp. $T^{\prime}$ ) be the universal cover of $\tilde{G} / \tilde{p}$ (resp. $\left.\tilde{G}^{\prime} / \tilde{p}^{\prime}\right)$. There is a $(k, c)$-quasi-isometry $h: P \rightarrow T$ (resp. $h^{\prime}: P^{\prime} \rightarrow T^{\prime}$ ) obtained by lifting $g$ to $\mathbb{H}^{2}$ and then restricting the domain. Let $h^{\prime *}: T^{\prime} \rightarrow P^{\prime}$ be a quasi-inverse of $h^{\prime}$. If $(\tilde{S}, \tilde{L})$ and $\left(\tilde{S}^{\prime}, \tilde{L}^{\prime}\right)$ are equivalent, then there is an isometry $i: P^{\prime} \rightarrow P$. Then the map $h i h^{\prime *}: T^{\prime} \rightarrow T$ is a $\left(k^{\prime}, c^{\prime}\right)$-quasi-isometry where $k^{\prime}$ and $c^{\prime}$ depend only on $k$ and $c$, and not on our choices of covers. By Lemma 3.3. we may assume that the edges of $G$, and hence also of $T$ and $T^{\prime}$, are longer than the constant $u\left(k^{\prime}, c^{\prime}\right)$ given by Lemma 3.1. Then $T$ and $T^{\prime}$ are isomorphic, contradicting the assumption that $(\tilde{G}, \tilde{p})$ and $\left(\tilde{G}^{\prime}, \tilde{p}^{\prime}\right)$ are inequivalent. So $(\widetilde{S}, \widetilde{L})$ and $\left(\widetilde{S}^{\prime}, \widetilde{L}^{\prime}\right)$ are inequivalent, and the result now follows from Lemma 3.2 


\section{Constructing immersed surfaces by gluing convex manifolds}

The material in this section is based on Thurston's bending deformation, and the treatment of this which is given in [1. We begin with a lemma about gluing convex manifolds. For a space $X$, we let $\stackrel{\circ}{X}$ denote the interior of $X$.

Lemma 4.1 Suppose that $X$ and $X^{\prime}$ are hyperbolic $n$-manifolds with convex boundary, and that $U, U^{\prime}$ are compact $n$-submanifolds, with an isometry $f$ : $U \rightarrow U^{\prime}$ such that

(1) $f(\partial U \cap \stackrel{\circ}{X}) \subset \partial X^{\prime}, f^{-1}\left(\partial U^{\prime} \cap \stackrel{\circ}{X}^{\prime}\right) \subset \partial X$, and

(2) $\partial X \cap f^{-1} \partial X^{\prime}$ is a non-empty subsurface of $\partial X$. Then the identification space $Y=X \cup_{U=f U} X^{\prime}$ is a hyperbolic manifold with convex boundary.

Proof Condition 1 guarantees that the identification space $Y$ is a manifold, with $\partial Y=(\partial X-U) \cup\left(\partial X^{\prime}-U^{\prime}\right) \cup\left(\partial X \cap f^{-1} \partial X^{\prime}\right)$. Condition 2 guarantees that $\partial X-U$ (resp. $\left.\partial X^{\prime}-U^{\prime}\right)$ can be extended to an open set $V \subset \partial Y$ (resp. $V^{\prime} \subset \partial Y$ ), such that $Y$ is locally convex at all points in $V$. Then $V, V^{\prime}$ and interior $\left(\partial X \cap f^{-1} \partial X^{\prime}\right)$ define an open covering of $\partial Y$ by convex sets, and so $Y$ is locally convex at all points in $\partial Y$.

If $W$ is a hyperbolic 3-manifold with convex boundary, let Core $(W)$ denote the convex core of $W$. This is the unique smallest convex sub-manifold of $W$ which carries $\pi_{1} W$. For any set $U$ in $W$, let $N_{\epsilon}(U)=\{x \in U \mid d(x, U) \leq \epsilon\}$.

Suppose $S$ is an orientable (possibly disconnected) hyperbolic surface with geodesic boundary, that $\ell_{i j} \subset \partial S(i=1,2, j=1, \ldots, n)$ are distinct boundary components, with orientations induced from $S$, and that length $\left(\ell_{1 j}\right)=\operatorname{length}\left(\ell_{2 j}\right)$ for all $j$. Let $L$ be the disjoint union of the $\ell_{i j}$ 's, and let $S(L)$ be the surface formed by identifying each $\ell_{1 j}$ and $\ell_{2 j}$ (preserving orientations) to a single loop, which we call $\ell_{j}$; we view $S(L)$ as a metric space, with path metric inherited from the $S_{i}$ 's. For each $j$, let $\theta_{j}$ be angle between 0 and $\pi$.

Lemma 4.2 There is a number $r=r\left(\theta_{1}, \ldots, \theta_{n}\right)>0$ such that, if $L$ has a collar neighborhood of radius $r$ in $S$, then there is a hyperbolic 3-manifold $W$, with convex boundary, such that:

(a) $W=\operatorname{Core}(W)$,

(b) $W \cong S(L) \times I$, and there is an isometric embedding $i: S(L) \rightarrow W$, such that $W$ retracts onto $i(S(L))$, 
(c) there is a regular neighborhood $N\left(\ell_{j}\right)$ of each $\ell_{i}$ in $S(L)$ so that $i\left(N\left(\ell_{j}\right)\right)$ is the union of a pair of geodesic annuli meeting at an angle $\theta_{j}$,

(d) for some $\epsilon>0$, the set $\mathcal{A}=\left\{i\left(\ell_{1}\right), \ldots, i\left(\ell_{n}\right)\right\}$ has the following geometric characterization:

$\mathcal{A}=\{$ infinite or closed geodesics $\gamma$ in $W \mid d(\gamma, F)>\epsilon$ for some component $F$ of $\partial W\}$.

Proof We will assume that $n=1$, the proof in the general case being similar. To reduce notation, let $\ell_{i}=\ell_{i 1}, \theta=\theta_{1}$, and $r=r_{1}$.

The outline of the construction is as follows. We first construct a hyperbolic solid torus $V$, which is a convex thickening of two annuli. We then construct a convex hyperbolic 3-manifold $Z$, by gluing together $S(L) \times I-N\left(\ell_{1}\right), S(L) \times$ $I-N\left(\ell_{2}\right)$ and $V$. Then finally we let $W=\operatorname{Core}(Z)$.

To construct the hyperbolic solid torus $V$, let $\epsilon>0$, let $\tilde{\ell}$ be a geodesic in $\mathbb{H}^{3}$, let $Q_{1}$ and $Q_{2}$ be geodesic half-planes in $\mathbb{H}^{3}$, with $\partial Q_{i}=\tilde{\ell}$, and let the angle of intersection of $Q_{1}$ and $Q_{2}$ be $\theta$. Let $Q=Q_{1} \cup Q_{2}$, let $B=N_{\epsilon}(Q)$, and let $H_{Q}$ be the convex component of $\mathbb{H}^{3}-Q$. For $p \in \partial N_{\epsilon}\left(Q_{i}\right)$, let $P_{p}$ be a hyperbolic plane such that $P_{p} \cap N_{\epsilon}\left(Q_{i}\right)=p$.

Claim There is an $r=r(\theta)$ such that, if $p \in \partial N_{\epsilon}\left(Q_{i}\right)$ and $d(p, \tilde{\ell})>r$, then $P_{p} \cap B=\{p\}$.

Proof of Claim There are two components of $\partial N_{\epsilon} Q_{i}-N_{\epsilon}(\tilde{\ell})$; one is contained in $H_{Q}$, which we denote $\partial^{-} N_{\epsilon}\left(Q_{i}\right)$, and the other is denoted $\partial^{+} N_{\epsilon}\left(Q_{i}\right)$.

Without loss of generality, let $p \in \partial N_{\epsilon}\left(Q_{1}\right)$. Suppose $p \in \partial^{+} N_{\epsilon}\left(Q_{1}\right)$. Let $P_{1}$ be the geodesic plane containing $Q_{1}$, and let $H_{1}$ be the half-space bounded by $P_{1}$ and containing $p$. If $r>\epsilon$, then $P_{p} \cap N_{\epsilon} Q_{1}=P_{p} \cap N_{\epsilon}\left(P_{1}\right)=\{p\}$, and so $P_{p} \subset H_{1}$. Since $N_{\epsilon}\left(Q_{2}\right) \cap H_{1} \subset N_{\epsilon}(\tilde{\ell})$, and since $d(p, \tilde{\ell})>\epsilon$, then $P_{p} \cap N_{\epsilon}\left(Q_{2}\right)=\emptyset$. Therefore $P_{p} \cap B=\{p\}$.

Suppose $p \in \partial^{-} N_{\epsilon}\left(Q_{1}\right)$. Let $Q^{\perp}$ be a hyperbolic plane which is orthogonal to $\tilde{\ell}$, and which contains $p$. Let $\alpha_{i}=Q_{i} \cap Q^{\perp}$, and let $\beta$ be a geodesic ray bisecting the $\alpha_{i}$ 's. Let $\delta(t)$ be a geodesic intersecting $\beta$ orthogonally at a distance $t$ from the endpoint of $\beta$ (see Figure 3).

Let $\partial^{ \pm} N_{\epsilon}\left(\alpha_{i}\right)=\partial^{ \pm} N_{\epsilon} Q_{i} \cap Q^{\perp}$. If $\delta(t) \cap B \neq \emptyset$, then by symmetry, $\delta(t)$ makes the same angle with both $\partial^{-} N_{\epsilon}\left(\alpha_{1}\right)$ and $\partial^{-} N_{\epsilon}\left(\alpha_{2}\right)$; call this angle $\psi(t)$. For small $t$, we have $\psi(t)>0$, and for large enough $t$, we have $\delta(t) \cap N_{\epsilon}\left(\alpha_{i}\right)=\emptyset$, so there must be some $t_{0}$ for which $\psi\left(t_{0}\right)=0$, and there is a corresponding 
geodesic $\delta\left(t_{0}\right)$ which is tangent to $B \cap Q^{\perp}$ at two points. There is then a hyperbolic plane, $Q\left(t_{0}\right)$, orthogonal to $Q^{\perp}$, which contains $\delta\left(t_{0}\right)$, and which is tangent to $B$ at two points.

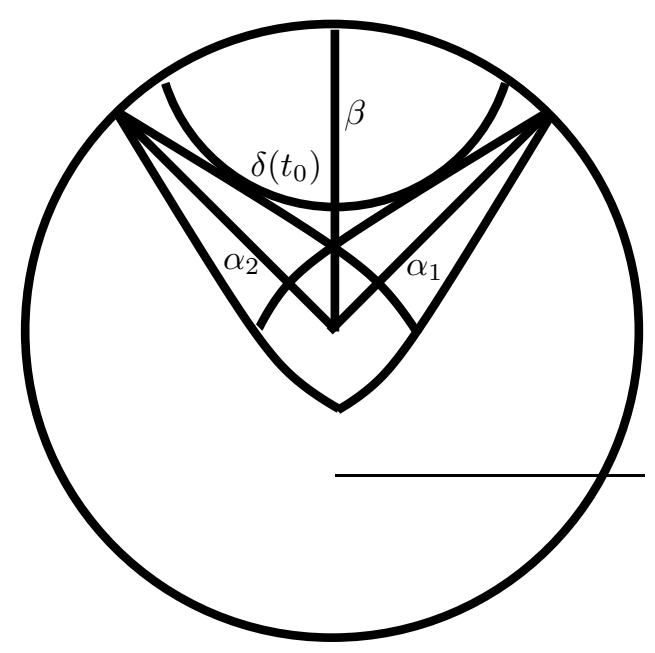

Figure 3: A convex thickening

Let $r=d\left(\tilde{\ell}, \delta\left(t_{0}\right) \cap B\right)$, and suppose $d(p, \tilde{\ell})>r$. Then $\delta\left(t_{0}\right)$ separates $p$ from $N_{\epsilon}\left(\alpha_{2}\right)$ in $Q^{\perp}-B$. Since the geodesic $P_{p} \cap Q^{\perp}$ can intersect $\delta\left(t_{0}\right)$ at most once, we must have $P_{p} \cap Q^{\perp} \cap N_{\epsilon}\left(\alpha_{2}\right)=\emptyset$, so $P_{p} \cap Q^{\perp} \cap B=\{p\}$. Let $H^{ \pm}$be the half-spaces in $\mathbb{H}^{3}$ bounded by $Q^{\perp}$. By symmetry, if $P_{p} \cap N_{\epsilon}\left(Q_{2}\right) \neq \emptyset$, then $P_{p} \cap H^{+} \cap N_{\epsilon}\left(Q_{2}\right)$ and $P_{p} \cap H^{-} \cap N_{\epsilon}\left(Q_{2}\right)$ are both non-empty, and therefore, by convexity, $P_{p} \cap Q^{\perp} \cap N_{\epsilon}\left(Q_{2}\right) \neq \emptyset$, which is a contradiction. Thus we conclude that $P_{p} \cap N_{\epsilon}\left(Q_{2}\right)=\emptyset$, so $P_{p} \cap B=\{p\}$, proving the claim.

Let $\operatorname{Hull}(B)$ denote the convex hull of $B$ in $\mathbb{H}^{3}$, which is the intersection of all closed half-spaces containing $B$. Let $\tau$ be a hyperbolic isometry (i.e. with real trace) in $\operatorname{Isom}^{+}\left(\mathbb{H}^{3}\right)$ such that $\tilde{\ell} / \tau$ is isometric to $\ell$. Since $\tau$ is hyperbolic, it preserves both $Q_{1}$ and $Q_{2}$, and hence also $\operatorname{Hull}(B)$. Let $s>r(\theta)$, and let $V=\left[\operatorname{Hull}(B) \cap N_{s}(\tilde{\ell})\right] / \tau$.

Let $S \times[-\epsilon, \epsilon]$ be a radius $\epsilon$ thickening of $S$ (more precisely, embed $\pi_{1} S$ as a Fuchsian subgroup of $\operatorname{Isom}\left(\mathbb{H}^{3}\right)$, embed $\tilde{S}$ in $\mathbb{H}^{3}$ isometrically as a $\pi_{1} S_{i^{-}}$ equivariant subset, and then define $\left.S \times[-\epsilon, \epsilon]=N_{\epsilon}(\tilde{S}) / \pi_{1} S\right)$. Let $U_{i}=(V-$ $\left.N_{r}(\tilde{\ell})\right) \cap\left(N_{\epsilon}\left(Q_{i}\right) / \tau\right)$, and let $U_{i}^{\prime}=S \times[-\epsilon, \epsilon] \cap\left(N_{s}\left(\ell_{i}\right)-N_{r}\left(\ell_{i}\right)\right)$.

By the claim, $\operatorname{Hull}(B)-N_{r}(\tilde{\ell})=B-N_{r}(\tilde{\ell})$, which implies that $U_{i}$ is isometric to $U_{i}^{\prime}$. By Lemma 4.1, we may form a hyperbolic manifold, $Z$, with convex 
boundary, from $(S \times[-\epsilon, \epsilon])-\left(N_{r}\left(\ell_{1}\right) \cup N_{r}\left(\ell_{2}\right)\right) \amalg V$ by gluing each $U_{i}$ to $U_{i}^{\prime}$ via isometry.

Since $Z$ has convex boundary, there is a unique smallest convex submanifold, $W=\operatorname{Core}(Z) \subset Z$, which is homotopy equivalent to $Z$. By the construction of $Z$, we have an isometric embedding $i: S(L) \rightarrow Z$, and $i(\ell)$ has a neighborhood in $Z$ which is a union of geodesic annuli meeting at an angle $\theta$. Since $i(S)$ is totally geodesic in $Z$, with geodesic boundary, we have that $i(S) \subset W$, and so $i(S(L)) \subset W$.

Since Core $\left(\pi_{1} W\right)$ is compact, then $\pi_{1} W$ is a quasi-Fuchsian surface group, and the only possibilities are that $W \cong S(L) \times I$ or that $W \cong S(L)$. Since $i(S(L))$ is not totally geodesic, $W \neq S(L)$, so $W \cong S(L) \times I$. Thus $W$ satisfies properties $a, b$ and $c$ of the lemma.

We now prove that $W$ satisfies property $d$. The surface $i(S(L))$ is an embedded copy of $S(L)$ in $W$, which separates $W$ into two components, $W^{+}$and $W^{-}$. Let $W^{-}$be the component which is convex in a neighborhood of $\ell$, and let $F=W^{-} \cap \partial W$.

The distance from $\ell$ to $F$ may be approximated to arbitrary precision by lengths of geodesic segments $\beta$ which are perpendicular to $\ell$. Let $\beta$ be such a segment, and let $\tilde{\beta}$ and $\tilde{\ell}$ be lifts to $\tilde{W} \subset \mathbb{H}^{3}$. Let $A_{1}, A_{2}$ be radius $r$, totally geodesic annuli in $S(L)$, with $A_{1} \cap A_{2}=\ell$, and let $\tilde{A}_{i} \subset \tilde{W}$ be a lift of $i\left(A_{i}\right)$ so that $\tilde{A}_{1} \cap \tilde{A}_{2}=\tilde{\ell}$. Let $Q^{\perp}$ be a geodesic plane containing $\tilde{\beta}$ and perpendicular to $\tilde{\ell}$, and let $q=Q^{\perp} \cap \tilde{\ell}$. Then $Q^{\perp} \cap \tilde{A}_{i}=\alpha_{i}$ is a geodesic segment of length at least $r$, and $\alpha_{1}$ and $\alpha_{2}$ meet at the point $q$ in an angle $\theta$. Let $T$ be the triangle with sides $\alpha_{1}$ and $\alpha_{2}$, and let $\delta$ be the third side of $T$. By convexity, $T \subset \tilde{W}$, and so length $(\beta) \geq d(q, \delta)$. Let $T^{\prime}$ be a triangle with two ideal vertices and regular vertex $q$, with angle $\theta$, and let $s(\theta)$ be the distance from $q$ to the opposite side of $T^{\prime}$. Then as $r$ becomes large, $d(q, \delta)$ approaches $s(\theta)$. Therefore $d(\ell, F) \approx \operatorname{length}(\beta) \approx s(\theta)$, and these approximations can be made arbitrarily close by increasing $r$. Therefore, after possibly increasing $r$ and decreasing $\epsilon$, we have that $d(\ell, F)>\epsilon$.

Let $\gamma$ be some other geodesic in $W$. Then $\gamma$ also represents a geodesic in $Z$. Since $V$ contains a unique closed geodesic, and no infinite geodesics, then $\gamma \cap(Z-V) \neq \emptyset$. However, $(Z-V) \cong(S(L) \times[-\epsilon, \epsilon])-N_{r}(\ell)$, and so $\gamma$ is within $\epsilon$ of both boundary components of $W$.

Next, some more notation. Suppose $f: S \rightarrow M$ is a totally geodesic immersion, and suppose $\ell \subset f(S)$ is a double curve, along which the angle of 
self-intersection is $\theta$. Let $\ell_{1}, \ell_{2} \subset S$ be two components of $f^{-1} \ell$, and let $L=\ell_{1} \cup \ell_{2}$.

Suppose that $\ell_{i}$ has a collar neighborhood of radius $r=r(\theta)$, where $r$ is the constant in Lemma 4.2 Let $N\left(\ell_{i}\right)$ be an embedded collar neighborhood of $\ell_{i}$, and let $\partial N\left(\ell_{i}\right)=\left\{\ell_{i 1}, \ell_{i 2}\right\}$. Further suppose, for convenience, that $L$ is non-separating. Let $S^{-}$be a compact surface homeomorphic to $S-\operatorname{int} N(L)$. The hyperbolic structure on $S$ induces a hyperbolic structure on $S^{-}$for which the components of $\partial S^{-}=\left\{\ell_{11}, \ell_{12}, \ell_{21}, \ell_{22}\right\}$ are all geodesics with the same length that $\ell$ has in $S$. Also, the orientation on $S_{i}$ induces an orientation on $\ell_{i j}$. Let $S(L)$ be the surface obtained from $S^{-}$by identifying $\ell_{1 j}$ and $\ell_{2 j}$, so that their orientations agree, and let $W=W(L, \theta) \cong S(L) \times I$ be the hyperbolic 3-manifold given by Lemma 4.2. The immersion $f: S \rightarrow M$ induces an immersion $f: S^{-} \rightarrow M$, which induces an immersion $g: S(L) \rightarrow M$, and extends to an immersion $g: W \rightarrow M$.

Lemma 4.3 If $\ell_{1}$ and $\ell_{2}$ have collar neighborhoods of radius $r(\theta)$ in $S$, then the immersion $g: S(L) \rightarrow M$ is $\pi_{1}$-injective.

Proof Since $W$ has convex boundary, the map $g: W \rightarrow M$ is $\pi_{1}$-injective, so $g: S(L) \rightarrow M$ is $\pi_{1}$-injective also.

Lemma 4.3 provides a way of constructing a great many $\pi_{1}$-injective immersions. For suppose $\tilde{S}$ is a cover of $S$, and suppose that $\ell$ has two (1-1) lifts, $\tilde{\ell}_{1}$ and $\tilde{\ell}_{2}$ in $\tilde{S}$. We say that the pair $\left(\tilde{S}, \tilde{L}=\tilde{\ell}_{1} \cup \tilde{\ell}_{2}\right)$ is admissable if the $\tilde{\ell}_{i}$ 's have collar neighborhoods of radius $r$ in $\tilde{S}$. If $(\tilde{S}, \tilde{L})$ is admissable, then by Lemma 4.3 the immersion $g: \tilde{S}(\tilde{L}) \rightarrow M$ is $\pi_{1}$-injective. Our goal is to show that different choices of admissable pairs will often result in inequivalent immersions; counting the number of choices for such pairs will then prove Theorem 1.2

Suppose, then, that $(\tilde{S}, \tilde{L})$ and $\left(\tilde{S}^{\prime}, \tilde{L}^{\prime}\right)$ are admissable pairs. Let $W$ be the convex thickening of $\tilde{S}(\tilde{L})$ provided by Lemma 4.2 with map $i: \tilde{S}(\tilde{L}) \rightarrow W$, and let $\ell=i(\tilde{L}) \subset W$. Let $P$ be the universal cover of $\widetilde{S}^{-}$.

We let $C$ be the universal cover of $W$; note that $C$ is isometric to the convex hull of the limit set of $g_{*} \pi_{1} \tilde{S}(\tilde{L})$. We also define analogous objects $W^{\prime}, i^{\prime}, \ell^{\prime}$, $\pi^{\prime}, \tilde{S}^{\prime-}, P^{\prime}$ and $C^{\prime}$ for $\left(\tilde{S}^{\prime}, \tilde{L}^{\prime}\right)$.

Recall from the previous section that the pairs $(\tilde{S}, \tilde{L})$ and $\left(\tilde{S}^{\prime}, \tilde{L}^{\prime}\right)$ are inequivalent if $P$ and $P^{\prime}$ are not isometric.

Lemma 4.4 If $(\tilde{S}, \tilde{L})$ and $\left(\tilde{S}^{\prime}, \tilde{L}^{\prime}\right)$ are admissable, inequivalent pairs, then $C$ and $C^{\prime}$ are not isometric. 
Proof Suppose $\phi: C \rightarrow C^{\prime}$ is an isometry. Identify $P$ with a component of the pre-image of $\widetilde{S}^{-}$in $C$. By Property d of Lemma $4.2 \phi\left(\pi^{-1} \ell\right)=\pi^{\prime-1} \ell^{\prime}$, so $\phi(P)$ is a totally geodesic surface in $C^{\prime}$, bounded by a subset of $\pi^{\prime-1} \ell^{\prime}$. We claim that $\phi(P)$ is isometric to $P^{\prime}$.

To see this, let $A$ be a separating, geodesic annulus embedded in $W^{\prime}$, containing $\ell^{\prime}$. Since $\phi(P) \cap \partial C=\emptyset$, each component of $\pi^{\prime-1} A \cap \phi(P)$ is an infinite geodesic; since each component of $\pi^{\prime-1} A$ contains a unique infinite geodesic, then each component of $\pi^{\prime-1} A \cap \phi(P)$ is a lift of $\ell^{\prime}$. Let $Q^{\prime}$ be the closure of a component of $\phi(P)-\pi^{\prime-1} \ell^{\prime}$, and let $\delta$ be a geodesic arc connecting two boundary components of $Q^{\prime}$. Since $\phi(P) \cap \pi^{\prime-1} \ell^{\prime}=\phi(P) \cap \pi^{\prime-1} A$, then $\pi^{\prime} \delta \subset W^{\prime}-A$, and since $W^{\prime}-A$ retracts onto the totally geodesic surface $i\left(\tilde{S}^{\prime}-\ell^{\prime}\right)$, and $\pi^{\prime} \delta$ is a geodesic, then $\pi^{\prime} \delta \subset i\left(\tilde{S}^{\prime-}\right)$. Therefore we may fix a copy of $P^{\prime}$ in $C^{\prime}$ which contains at least two boundary components of $Q^{\prime}$. This implies that $P^{\prime}$ and $Q^{\prime}$ are contained in the same geodesic plane $H$ in $C^{\prime}$. Both $P^{\prime}$ and $Q^{\prime}$ are the closure of the unique component of $H-\pi^{\prime-1} \ell^{\prime}$ which contains $\stackrel{\circ}{\delta}$, and therefore $P^{\prime}=Q^{\prime}$.

Finally, if $R^{\prime}$ is any other component of $\phi(P)-\pi^{\prime-1}\left(\ell^{\prime}\right)$, then $\pi^{\prime} P^{\prime}=\pi^{\prime} Q^{\prime}=$ $\pi^{\prime} R^{\prime}=\tilde{S}^{\prime-}$, which forces $Q^{\prime}$ and $R^{\prime}$ to meet at an angle $\theta$, contradicting the fact that $\phi(P)$ is totally geodesic. Therefore $\phi(P)=Q^{\prime}=P^{\prime}$.

Proof (Of Theorem 1.2) We have already proved the upper bound, and we now prove the lower bound. We are given a totally geodesic immersion of a hyperbolic surface $f: S \rightarrow M$, with a loop of transverse intersection $\ell$, at an angle $\theta$. Let $r=r(\theta)$ be the constant given by Lemma 4.2 Let $\ell_{1}$ and $\ell_{2}$ be components of $f^{-1} \ell$, and let $L=\left\{\ell_{1} \cup \ell_{2}\right\}$. Since $\pi_{1} S$ is LERF (by [7]), there is a finite cover $\pi: \tilde{S} \rightarrow S$ in which $L$ lifts to a non-separating curve with collar neighborhood of radius $r$. Replacing the immersion $f: S \rightarrow M$ with the immersion $f \pi: \tilde{S} \rightarrow M$, we may assume that $L$ is non-separating, with a collar neighborhood of radius at least $r$. Then any further cover $(\tilde{S}, \tilde{L})$ of $(S, L)$ (where $\tilde{L}$ is a $1-1$ lift of $L$ ) is admissable.

By Lemma 3.4 the number of admissable, inequivalent covers $(\tilde{S}, \tilde{L})$ of $(S, L)$ grows factorially in the covering degree of $\tilde{S}$, which is proportional to the genus of the resulting immersed surfaces. Since the pairs are admissable, the corresponding immersions are $\pi_{1}$-injective by Lemma 4.3. Since the pairs are inequivalent, Lemma 4.4 implies that the corresponding subgroups of $\pi_{1} M$ are inequivalent under the relation generated by commensurability and conjugacy. Theorem 1.2 follows. 
Remark The proof actually shows that the surface subgroups are inequivalent under the relation generated by commensurability and conjugacy in $P S L_{2}(C)$.

\section{Example: Reflection orbifolds}

Now we consider the special case of right-angled reflection orbifolds. These always have transversely immersed, totally geodesic surfaces (corresponding to any pair of adjacent faces) and so Theorem 1.2 applies. However, in this case there are combinatorial proofs which give more explicit information.

We first give a combinatorial construction of immersed incompressible surfaces in right-angled reflection orbifolds. This is a well-known construction, and the treatment here is similar to that of [1]. We will need the following definitions and notation.

Let $P$ be a right-angled polyhedron, let $\Gamma(P)$ be the group generated by reflections in the faces of $P$, and let $\Gamma^{+}(P)$ denote the subgroup of orientationpreserving elements in $P$. We define a face disk of $P$ to be a connected and simply connected union of faces of $P$, and a face loop on $P$ to be a collection of $n$ faces $F_{i}$ such that $F_{i}$ is adjacent to $F_{j}$ if and only if $|i-j|=1(\bmod n)$. Let $D$ be a face disk of $P$. We say that $D$ satifies the convexity condition if the faces of $P$ transverse to $D$ form a face loop (see Figure 4 ).

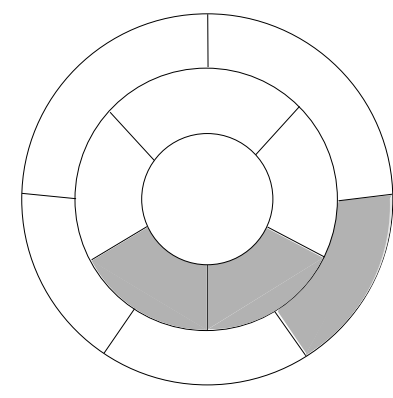

(a)

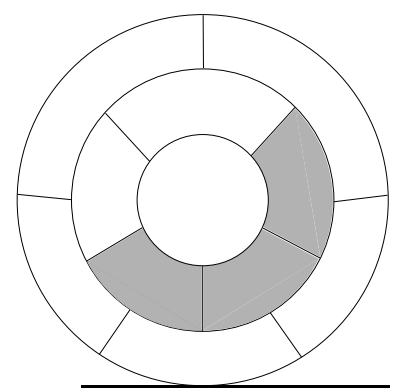

(b)

Figure 4: (a) A face disk satisfying the convexity condition (b) A face disk which does not satisfy the convexity condition

If $F$ is a face in $P$, we let $\rho_{F}: \mathbb{H}^{3} \rightarrow \mathbb{H}^{3}$ denote reflection in the plane containing $F$. If $X$ and $Y$ are spaces, and $\rho$ is a homeomorphism from a subset of $X$ to a subset of $Y$, let $X \cup_{\rho} Y$ denote $X$ glued to $Y$ via the gluing map $\rho$. 
The incompressibility results of this section are essentially an elaboration of the following elementary fact: given a collection of planes in $\mathbb{H}^{3}$, each meeting the next at right angles, and such that non-consecutive planes are disjoint, then the group generated by their reflections is isomorphic to the obvious 2-dimensional reflection group.

Lemma 5.1 Suppose that $P_{1}$ and $P_{2}$ are right-angled polyhedra in $\mathbb{H}^{3}$, that $D_{i} \subset P_{i}$ are face disks satisfying the convexity condition, and that $F_{i} \subset P_{i}$ are faces transverse to $D_{i}$, with $D_{i} \cap F_{i}=e_{i 1}, \ldots, e_{i m}$. Suppose there is an orientation-reversing isometry $\rho: F_{1} \rightarrow F_{2}$ such that $\rho\left(F_{1}, e_{11}, \ldots, e_{1 m}\right)=$ $\left(F_{2}, e_{21}, \ldots, e_{2 m}\right)$. Then $D_{1} \cup_{\rho} D_{2}$ satisfies the convexity condition in $P_{1} \cup_{\rho} P_{2}$.

Proof Since $P_{i}$ are right-angled polyhedra, $P_{1} \cup_{\rho} P_{2}$ is a right-angled polyhedron also.

The lemma then follows from the easily verified fact that the amalgamation of two face loops along a common face is again a face loop.

Since $D$ abuts the reflection planes of $P$ in right angles, it defines a closed 2-orbifold $\bar{D}$ in $\mathbb{H}^{3} / \Gamma(P)$ by making the edges of $D$ into reflectors. The image of $\pi_{1} \bar{D}$ in $\Gamma$ is generated by the reflections in the faces of $P$ which $D$ interesects transversely. The following result about the incompressibility of $\pi_{1} \bar{D}$ is a reformulation of a well-known property of Coxeter groups.

Proposition 5.2 If $D$ satisfies the convexity condition, then the fundamental group of $\bar{D}$ injects into $\Gamma(P)$.

Proof We fix a copy of $D$ and $P$ in $\mathbb{H}^{3}$. It will suffice to show that the union of $D$ with its translates under $\pi_{1}(\bar{D})$ forms an embedded disk.

Since $\bar{D}$ is a right-angled reflection orbifold, it may be developed in $\mathbb{H}^{3}$ by successively doubling $(P, D)$ across the faces of $P$ which $D$ intersects transversely.

Let $F$ be a face of $P$ which $D$ intersects transversely, and let $P_{2}=P \cup \rho_{F} P$. Then $D$ develops in $P_{2}$ as $D \cup \rho_{F} D$. By Lemma 5.1 $D \cup \rho_{F} D$ satisfies the convexity condition in $P \cup \rho_{F} P$. In particular, $D \cup \rho_{F} D$ is a disk.

Iterating this process, we obtain after each doubling an embedded disk. It follows that the union of $D$ with all its translates is embedded and simply connected. 
Suppose that $\widehat{P} \subset \mathbb{H}^{3}$ is a right-angled polyhedron which is made up of copies of $P$, and that $D$ is a face disk in $\widehat{P}$ satisfying the convexity condition. By Theorem [5.2, the reflection orbifold $\bar{D}$ is incompressible in $\mathbb{H}^{3} / \Gamma(\widehat{P})$, so under the covering map $\pi: \mathbb{H}^{3} / \Gamma(\widehat{P}) \rightarrow \mathbb{H}^{3} / \Gamma(P)$, it projects to an immersed incompressible 2-orbifold $\pi \bar{D}$. As we did in Section 3 , we can cut and paste $\pi \bar{D}$, to create many different immersions of 2-orbifolds in $\mathbb{H}^{3} / \Gamma(P)$.

Let $e_{1}, e_{2} \subset \partial D$ be edges of $\widehat{P}$, let $F_{i}$ be the faces adjacent to $e_{i}$ in $D$, and let $F_{i}^{\prime}$ be the face adjacent to $e_{i}$ in $\widehat{P}-D$. Suppose there is an orientationpreserving element $\gamma \in \Gamma(P)$ such that $\gamma\left(F_{1}, e_{1}\right)=\left(F_{2}, e_{2}\right)$. We "cut" $\bar{D}$ by first making $e_{1}, e_{2}$ into boundary edges (not reflector edges); call this orbifold $\bar{D}^{\prime}$. We then "paste" by forming the quotient orbifold $\bar{D}^{\prime} / \rho_{F_{2}^{\prime}} \gamma$. Since $\rho_{F_{2}} \gamma$ takes $e_{1}$ to $e_{2}$, this is a closed 2-orbifold, and since $\rho_{F_{2}} \gamma \in \Gamma(P)$, there is a natural map from $\bar{D}^{\prime} / \rho_{F_{2}} \gamma$ into $\mathbb{H}^{3} / \Gamma(P)$.

We repeat this procedure at different edges of $D$, and encode the collection of gluings by an order 2 permutation $\sigma$, defined on the edges of $D$. Let $D_{\sigma}$ denote the immersed, closed 2-orbifold in $\mathbb{H}^{3} / \Gamma(P)$ obtained by cutting and pasting $\pi \bar{D}$ according to the permutation $\sigma$, and let $f_{\sigma}: D_{\sigma} \rightarrow \mathbb{H}^{3} / \Gamma(P)$ be the associated immersion. We define the orbifold degree of a vertex $v$ of $D_{\sigma}$ to be the degree of $v$ in the universal cover of $D_{\sigma}$.

Proposition 5.3 If $D$ satisfies the convexity condition, and if each vertex of $D_{\sigma}$ has (orbifold) degree 4 , then the immersion $f_{\sigma}: D_{\sigma} \rightarrow \mathbb{H}^{3} / \Gamma(P)$ is $\pi_{1}$-injective.

Proof The proof is similar to the proof of Theorem [5.2. We fix a copy of $\widehat{P}$ and $D$ in $\mathbb{H}^{3}$ and show that the translates of $D$ under $\pi_{1}\left(D_{\sigma}\right)$ form an embedded disk.

The translates of $D$ under $\pi_{1}\left(D_{\sigma}\right)$ may be obtained by successively doubling $\widehat{P}$ via the various symmetries in $\Gamma(P)$ which correspond to the permutation $\sigma$. After the first doubling, then, $D$ is glued to $\rho D$ along some edge, via some $\rho \in \Gamma(P)$. The faces $D$ and $\rho D$ satisfy the convexity condition, in $\widehat{P}$ and $\rho \widehat{P}$ respectively, so by Lemma 5.1 their union satisfies the convexity condition in $\widehat{P} \cup \rho \widehat{P}$.

Doubling again along a face adjacent to the initial doubling face, we get four copies of $D$ around a vertex, which by the vertex condition will glue appropriately. Again, by Lemma [5.1, this union will be a face disk satisfying the convexity condition.

Iterating this process, we obtain after each doubling an embedded disk, and therefore the union of $D$ with all its translates is an embedded disk. 
We now give an explicit construction of a family of inequivalent immersed surfaces in a right-angled reflection orbifold.

We are given a right-angled polyhedron $P$. Compact Coxeter polyhedra in $\mathbb{H}^{3}$ have vertices of degree three, and no faces with fewer than five edges; an Euler characteristic computation then shows that every Coxeter polyhedron has a face with exactly five edges.

Let $F_{1}$ be a face of $P$ with five edges. We paste together $n$ copies of $F_{1}$, and attach one additional adjacent face $F_{2}$, as indicated in Figure 5 , the resulting complex, which we call $D$, is a face disk for a right-angled Coxeter polyhedron $\widehat{P}$ made up of $n$ copies of $P$. We cut and paste the reflection orbifold $\bar{D}$ along alternating edges of $D$, as shown in Figure 5. Associated to any order 2 permutation, $\sigma$, of these edges there is an edge pairing, and a resulting 2orbifold $D_{\sigma}$, with an immersion $f_{\sigma}: D_{\sigma} \rightarrow \mathbb{H}^{3} / \Gamma(P)$.

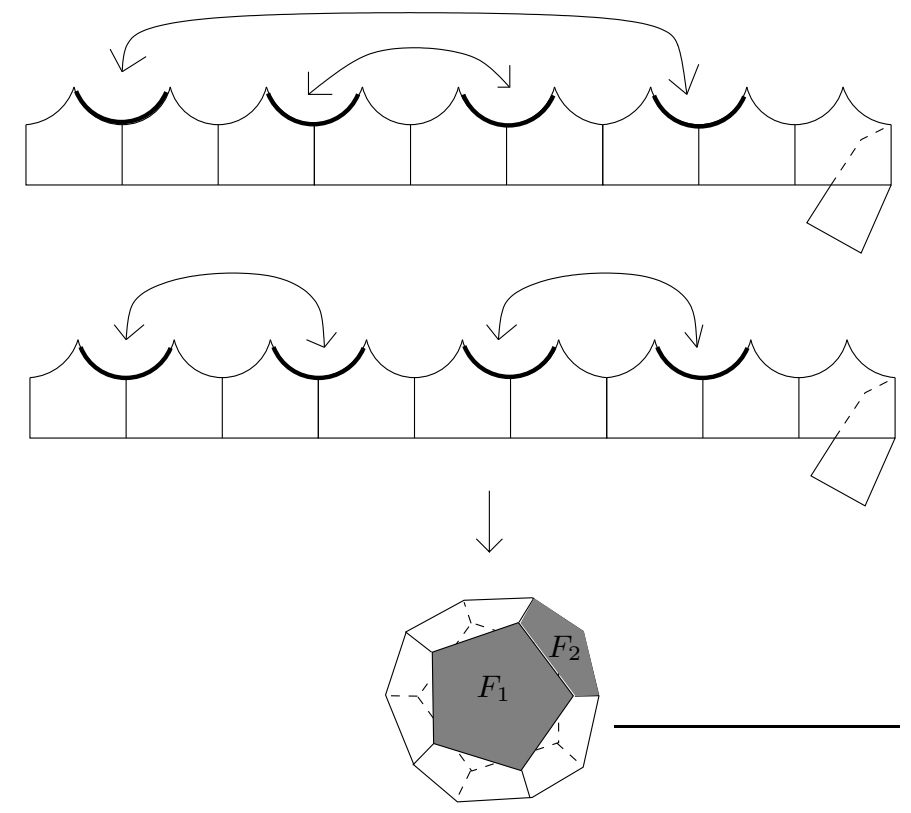

Figure 5: Different gluings will result in inequivalent surfaces

Lemma 5.4 The immersion $f_{\sigma}: D_{\sigma} \rightarrow \mathbb{H}^{3} / \Gamma(P)$ is $\pi_{1}$-injective.

Proof The union of two adjacent faces of a compact Coxeter polyhedron always satisfies the convexity condition, since such a polyhedron cannot have face loops of length less than five. Therefore $D$ satisfies the convexity condition. 
Also, since $\sigma$ has order two, the vertices of $D_{\sigma}$ all have orbifold degree four, and so the lemma follows from Proposition 5.3 .

The resulting immersions are in fact inequivalent. The key point is that we have control over the limit sets.

Let $\tilde{P}$ be a fixed lift of $P$ to $\mathbb{H}^{3}$, and let $\tilde{D} \subset \tilde{P}$ be a fixed lift of $D$, which determines a fixed map $f_{\sigma *} \pi_{1} D_{\sigma} \rightarrow \Gamma(P)$. Let $\Lambda\left(f_{\sigma *} \pi_{1} D_{\sigma}\right)$ denote the limit set of $f_{\sigma *} \pi_{1} D_{\sigma}$ in $\mathbb{H}^{3}$. Let $\mathcal{H}=\left\{H_{1}, \ldots, H_{n}\right\} \subset \mathbb{H}^{3}$ denote the half-spaces which intersect $\tilde{P}$ in faces orthogonal to $\tilde{D}$; we label these so that $H_{i} \cap H_{i+1} \neq \emptyset$, and $H_{i} \cap H_{j}=\emptyset$ if $|i-j| \geq 2(\bmod n)$. Then we have:

\section{Lemma 5.5}

(1) $\Lambda\left(f_{\sigma *} \pi_{1} D_{\sigma}\right) \cap H_{i} \neq \emptyset$ for all $i$.

(2) $\Lambda\left(f_{\sigma *} \pi_{1} D_{\sigma}\right) \subset \bigcup_{i=1}^{n} H_{i}$.

Proof Each $H_{i}$ is stabilized by $\left\langle\rho_{H_{i-1}}, \rho_{H_{i+1}}>\right.$. Since $H_{i-1}$ and $H_{i+1}$ are non-adjacent, this group is infinite, and so it has a limit point in $H_{i}$, proving 1.

Since every point in $\Lambda\left(f_{\sigma *} \pi_{1} D_{\sigma}\right)$ is a limit of a sequence of points in $\tilde{D}_{\sigma}$, then to prove 2 , it is enough to show that $\tilde{D}_{\sigma} \subset \bigcup_{i=1}^{n} H_{i} \cup \tilde{D}$.

As in the proof of Lemma [5.3, $\tilde{D}_{\sigma}$ may be constructed by successive doublings. More precisely, there is a sequence of Coxeter polyhedra $\tilde{P}=P_{1}, P_{2}, \ldots$, and face disks $D_{i} \subset P_{i}$, such that $\left(P_{i+1}, D_{i+1}\right)=\left(P_{i}, D_{i}\right) \cup\left(\gamma_{i} P_{i}, \gamma_{i} D_{i}\right)$, for some $\gamma_{i} \in f_{\sigma *} \pi_{1} D_{\sigma}$, and $\tilde{D}_{\sigma}=\bigcup_{i} D_{i}$. Let $\mathcal{Q}_{i}$ be the set of hyperbolic planes of $P_{i}$ which are transverse to $D_{i}$.

Claim For every $i$, if $Q \in \mathcal{Q}_{i}$, then $Q \subset \bigcup_{H_{j} \in \mathcal{H}} H_{j}$.

Proof of claim The statement is true for $i=1$, and suppose it is true for $i=n$. Let $J_{n}$ be the plane fixed by $\gamma_{n}$, and suppose without loss of generality that $J_{n} \subset H_{1}$. All of the planes in $\mathcal{Q}_{n+1}-\mathcal{Q}_{n}$ correspond to faces of $\rho_{n} P_{n}$ which are not adjacent to the face containing $J_{n}$. Since $\gamma_{n} P_{n}$ is a Coxeter polyhedron, these planes are disjoint from $J_{n}$, and therefore $J_{n}$ separates every plane in $\mathcal{Q}_{n+1}-\mathcal{Q}_{n}$ from $\tilde{D}$. Therefore every plane in $\mathcal{Q}_{n+1}-\mathcal{Q}_{n}$ is contained in $H_{1}$, and so every plane in $\mathcal{Q}_{n+1}$ is contained in some $H_{j}$. The claim follows by induction.

From the claim it follows that $D_{j} \subset \bigcup_{i} H_{i}$ for all $j$, and so $\tilde{D}_{\sigma} \subset \bigcup_{i} H_{i}$, as required. 
Let $\sigma$ and $\sigma^{\prime}$ be distinct order two permutations. We choose arbitrary lifts $\tilde{D}$ and $\tilde{D}^{\prime}$ of $D$ in $\mathbb{H}^{3}$, and these determine maps $f_{\sigma *}: \pi_{1} D_{\sigma} \rightarrow \Gamma(P)$ and $f_{\sigma^{\prime} *}: \pi_{1} D_{\sigma^{\prime}} \rightarrow \Gamma(P)$. Let $\Gamma=f_{\sigma *} \pi_{1} D_{\sigma}$, and $\Gamma^{\prime}=f_{\sigma / *} \pi_{1} D_{\sigma^{\prime}}$.

Proposition 5.6 No conjugate of $\Gamma$ is commensurable with $\Gamma^{\prime}$ in $\Gamma(P)$.

Before proving this, we need one further lemma. Let $A, B \subset \mathbb{H}^{3}$, and let $\mathcal{Q}$ be the collection of all the geodesic planes in $\mathbb{H}^{3}$ which project to $\partial P$. Define the combinatorial distance $d_{P}(A, B)$ to be the number of planes in $\mathcal{Q}$ which separate $A$ and $B$. Let $\mathcal{H}=\left\{H_{1}, \ldots, H_{k}\right\}$ be the half spaces perpendicular to $\partial \tilde{D}$. For each $H_{i}$, there is an element $\gamma_{i} \in \Gamma$ such that $\gamma_{i} \tilde{D} \cap \tilde{D} \subset \partial H_{i}$.

Lemma 5.7 Suppose $A \subset \mathbb{H}^{3}$, and that $H_{i} \supset A$. Then $d_{P}\left(\gamma_{i} \tilde{D}, A\right)<$ $d_{P}(\tilde{D}, A)$.

Proof Otherwise there is a plane $Q \in \mathcal{Q}$ which separates $\gamma_{i} \tilde{D}$ from $A$ but does not separate $\tilde{D}$ from $A$. Then $Q$ must separate a subset of $\tilde{D}$ from $\gamma_{i} \tilde{D}$, and so $Q \cap \stackrel{\check{D}}{D} \neq \emptyset$. Also, $Q \neq \partial H_{i}$, and so there is a $j$ such that $Q, \partial H_{i}$, and $\partial H_{j}$ all intersect, which forces $Q$ to be adjacent to $H_{i}$, contradicting the assumption that $Q \cap \stackrel{\check{D}}{0} \neq \emptyset$.

Proof of Proposition 5 5.6 Since $\Gamma$ and $\Gamma^{\prime}$ were chosen only up to an arbitrary conjugation (depending on the choice of lifts $\tilde{D}$ and $\tilde{D}^{\prime}$ ), it is enough to show that $\Gamma$ is not commensurable with $\Gamma^{\prime}$, and for this, it is enough to show that $\Lambda(\Gamma) \neq \Lambda\left(\Gamma^{\prime}\right)$.

Suppose that $\Lambda(\Gamma)=\Lambda\left(\Gamma^{\prime}\right)$, and suppose also that $\tilde{D} \neq \tilde{D}^{\prime}$. Let $\mathcal{H}=$ $\left\{H_{1}, \ldots, H_{k}\right\}$ and $\mathcal{H}^{\prime}=\left\{H_{1}^{\prime}, \ldots, H_{k}^{\prime}\right\}$ be the half spaces perpendicular to $\partial \tilde{D}$ and $\partial \tilde{D}^{\prime}$, let $\gamma_{i} \in \Gamma$ such that $\gamma_{i} \tilde{D} \cap \tilde{D} \subset \partial H_{i}$, and define $\gamma_{i}^{\prime}$ similarly. Then Lemma 5.5 implies that every $H_{i}^{\prime}$ is contained in some $H_{j}$, and in particular $\tilde{D}^{\prime} \subset H_{j}$ for some $j$. By Lemma 5.7 $d_{P}\left(\gamma_{j} \tilde{D}, \tilde{D}^{\prime}\right)<d_{P}\left(\tilde{D}, \tilde{D}^{\prime}\right)$, and we may replace $\tilde{D}$ by $\gamma_{j} \tilde{D}$, without affecting $\Lambda(\Gamma)$. Eventually, we have $d_{P}\left(\tilde{D}, \tilde{D}^{\prime}\right)=0$, so $\tilde{D}$ and $\tilde{D}^{\prime}$ intersect in some lift of $P$.

Recall $\tilde{D}$ and $\tilde{D}^{\prime}$ both consist of $n$ lifts of $F_{1}$ and a single lift of $F_{2}$. If $\tilde{D}$ and $\tilde{D}^{\prime}$ overlap on some lift of $F_{1}$ and differ on the lift of $F_{2}$, then there is some $H_{j}$ (adjacent to the lift of $F_{2}$ ) which is disjoint from every $H_{i}^{\prime}$, contradicting Lemma 5.5. So $\tilde{D}$ and $\tilde{D}^{\prime}$ contain the same lift of $F_{2}$, and it then follows that $\tilde{D}$ and $\tilde{D}^{\prime}$ co-incide. 
We now have $\tilde{D}=\tilde{D}^{\prime}$, but by assumption, the gluings $\sigma$ and $\sigma^{\prime}$ disagree on some edge, which lifts to some $H_{i}$. Then $\mathcal{F}=\tilde{D} \cup \gamma_{i} \tilde{D}$ and $\mathcal{F}^{\prime}=\tilde{D} \cup \gamma_{i}^{\prime} \tilde{D}$ are face disks in a Coxeter polyhedron $\widehat{P}$ (made of $2 n$ copies of $\tilde{P}$ ) which satisfy the convexity condition. Let $\mathcal{H}$ and $\mathcal{H}^{\prime}$ be the sets of half-spaces transverse to $\mathcal{F}$ and $\mathcal{F}^{\prime}$. Since $\sigma$ and $\sigma^{\prime}$ disagree, there is an $H_{i} \in \mathcal{H}$ which is disjoint from every $H_{j} \in \mathcal{H}^{\prime}$. Lemma 5.5 then shows that $\Lambda(\Gamma) \neq \Lambda\left(\Gamma^{\prime}\right)$, which is a contradiction.

The lower bound given in Theorem 1.3 can be computed from Proposition [5.6 as follows: first we must modify the construction of $D_{\sigma}$ to produce closed, non-singular surfaces, instead of 2-orbifolds. This can be done by starting with 4 copies of $F_{1}$ and $4 n$ copies of $F_{2}$, which can be glued into a closed surface. There $4((n-1) / 2)=2 n-2$ edges (corresponding to the dark edges of Figure 5) along which we may cut and paste via an arbitrary transposition $\sigma$, to get an immersion of a closed surface $S_{\sigma}$. By the same method as the proof of Proposition 5.6, it can be shown that different choices of $\sigma$ result in inequivalent, $\pi_{1}$-injective immersions.

The Euler characteristic of $S_{\sigma}$ is $-n-c$, for some fixed constant $c$ depending on the number of sides of $F_{2}$, and there are at least $(n-1)$ ! choices of transpositions $\sigma$ resulting in inequivalent immersions. We have $g=(n+c) / 2+1$, so $n-1=$ $2 g-c-3$, and so $s_{1}(M, g)>(2 g-c-3) !>e^{g \log g}$, for large $g$.

Finally, we give a combinatorial proof of the upper bound in this setting. The first step is to show that every incompressible immersed surface in a reflection orbifold can be homotoped to a standard form.

We view $\mathbb{H}^{3} / \Gamma(P)$ as an orbifold, with an underlying polyhedral complex determined by $P$.

Lemma 5.8 Let $S$ be a closed surface of positive genus, and let $\Gamma \subset \Gamma(P)$, with $\Gamma \cong \pi_{1} S$. Then there is a polyhedral 2-complex $K$ on $S$, and a immersion $f: S \rightarrow \mathbb{H}^{3} / \Gamma(P)$, which is cellular with respect to $K$ and $P$, such that $f_{*}\left(\pi_{1} S\right)=\Gamma$, and such that the universal cover of $S$ lifts to an embedded plane in $\mathbb{H}^{3}$.

Proof There is a unique cover $\pi: M \rightarrow \mathbb{H}^{3} / \Gamma(P)$ such that $\operatorname{Image}\left(\pi_{*}\right)=\Gamma$. The pre-image of $P$ in $M$ defines a polyhedral complex $K(P)$ on $M$. By Scott's compact core theorem, $M$ has a compact core $M^{-}$, such that $\pi_{1}\left(M^{-}\right) \cong \Gamma$, and Waldhausen's theorems, $M^{-} \cong S \times I$. Let $K(P)^{-}$denote the polyhedra of $K(P)$ which intersect $M^{-}$. There is an embedding $i: S \rightarrow \partial K\left(P^{-}\right)$, and 
the polyhedral structure of $\partial K\left(P^{-}\right)$pulls back to a polyhedral structure $K$ on $S$. Then $\pi i: S \rightarrow \mathbb{H}^{3} / \Gamma(P)$ is the desired immersion.

Let $\tilde{P}$ be a lift of $P$ to $\mathbb{H}^{3}$. A sub-complex $\mathcal{F}$ of the 2 -skeleton of $K$ is a face disk if $\left.f\right|_{\mathcal{F}}$ lifts to a face disk of $\tilde{P}$. If $v$ is a vertex of $\partial \mathcal{F}, \operatorname{deg}_{K}(v)$ denotes the degree of $v$ in $K$, whereas $\operatorname{deg}_{\mathcal{F}}(v)$ denotes the degree of $v$ in $\mathcal{F}$. Let $\mathcal{V}(\mathcal{F}, i)=\left\{v \in \partial \mathcal{F} \mid \operatorname{deg}_{\mathcal{F}}(v)=i\right\}$.

We require the following general fact about compact Coxeter polyhedra in $\mathbb{H}^{3}$.

Lemma 5.9 Let $\mathcal{F}$ be a face disk of $P$. If $|\mathcal{V}(\mathcal{F}, 2)| \leq 4$, then $\mathcal{F}$ contains more than half of the faces of $P$.

Proof Let $\mathcal{F}^{\prime}=P-\mathcal{F}$. If $|\mathcal{V}(\mathcal{F}, 2)|=0$, then $\mathcal{F}^{\prime}$ is a single face. If $|\mathcal{V}(\mathcal{F}, 2)|=$ 1 , then $\left|\mathcal{V}\left(\mathcal{F}^{\prime}, 3\right)\right|=1$, which implies the existence of an edge in $P$ which is adjacent to only one face, which is impossible. If $|\mathcal{V}(\mathcal{F}, 2)|=2$, then $\mathcal{F}^{\prime}$ consists of exactly two faces of $P$. If $|\mathcal{V}(\mathcal{F}, 2)|=3$, then $\mathcal{F}^{\prime}$ consists of three faces meeting at a common vertex. If $|\mathcal{V}(\mathcal{F}, 2)|=4$, then, adjacent to its boundary $\mathcal{F}^{\prime}$ contains faces $F_{1}, \ldots, F_{4}$, with $F_{i} \cap F_{i+1} \neq \emptyset(\bmod 4)$. Since $P$ is a rightangled, compact Coxeter polyhedron, it contains no face loops of length three or four, and no vertices of degree four, and so this is impossible.

We conclude that $\mathcal{F}^{\prime}$ contains at most three faces. Since a compact, rightangled Coxeter polyhedron in $\mathbb{H}^{3}$ must contain at least twelve faces, the result follows.

A face disk $\mathcal{F}$ of $K$ is special if

(1) $\operatorname{deg}_{K}(v) \geq 4$ for all $v \in \partial \mathcal{F}$, and

(2) $|\mathcal{V}(\mathcal{F}, 2)| \geq 5$.

Lemma 5.10 There is a choice of $f$ and $K$ as in Lemma 5.8, such that $K$ has a partition into special face disks.

We say that the resulting immersed surface $S$ is in standard form. For a complex $K$, let $K^{(i)}$ denote the $i$-skeleton, and let $\left|K^{(i)}\right|$ denote the cardinality of $K^{(i)}$.

Proof Let $f$ and $K$ be as given by Lemma 5.8. If $F_{1}, F_{2}$ are in $K^{(2)}$, say that $F_{1} \equiv F_{2}$ if there is a vertex $v \in F_{1} \cap F_{2}$ with $\operatorname{deg}_{K}(v)=3$. Let $\mathcal{F}_{1}, \ldots, \mathcal{F}_{n}$ be the equivalence classes of $\equiv$. Every $\mathcal{F}_{i}$ lifts to a single copy of $P$ in $\mathbb{H}^{3}$, and so $\mathcal{F}_{i}$ is a face disk for all $i$. 
Suppose $\left|\mathcal{V}\left(\mathcal{F}_{i}, 2\right)\right| \leq 4$ for some $i$. Then we enlarge $\mathcal{F}_{i}$ to a maximal face disk, by adding as many faces of $K^{(2)}$ as possible. Let $\mathcal{F}_{i}^{\prime}$ denote $\partial P-f\left(\mathcal{F}_{i}\right)$. Then $\left.f\right|_{\mathcal{F}}$ can be homotoped rel. boundary, and the polyhedral structure on $S$ can be changed, so that $f\left(\mathcal{F}_{i}\right)=\mathcal{F}_{i}^{\prime}$, and $f$ remains a cellular immersion. Also, when lifted to the universal cover, this homotopy is supported on a single copy of $P$, and one may check that the lift of $f$ remains an embedding. We then obtain new equivalence classes, and repeat the process. By Lemma 5.9 every step reduces $\left|K^{(2)}\right|$, so the process must eventually terminate in a special complex.

Suppose now that $f: K \rightarrow \mathbb{H}^{3} / \Gamma(P)$ is as given by Lemma 5.10. Let $K^{\prime}$ be the corresponding complex of special face disks.

Lemma 5.11 If $v$ is a vertex of $K$ with $\operatorname{deg}_{K}(v) \leq 5$, then $\operatorname{deg}_{K^{\prime}}(v) \geq 4$.

Proof Suppose $\operatorname{deg}_{K^{\prime}}(v) \leq 3$. Let $\mathcal{F}_{i}, i=1, \ldots, n \leq 3$ be the face disks of $K^{\prime}$ which are adjacent to $v$. Lift $f$ to an embedding $\tilde{f}: \tilde{S} \rightarrow \mathbb{H}^{3}$, choose a lift $\tilde{v}$ of $v$, and let $\tilde{\mathcal{F}}_{i}$ be the lift of $\mathcal{F}_{i}$ adjacent to $v$. Then $\tilde{f} \tilde{\mathcal{F}}_{i}$ is a face disk of some lift of $P$. Since $P$ is a right-angled polyhedron, then in a neighborhood of $\tilde{v}$, the tesellation of $\mathbb{H}^{3}$ is equivalent to the coordinate planes of $\mathbb{R}^{3}$. Since $\tilde{f} \tilde{\mathcal{F}}_{i}$ is a face disk, then in a neighborhood of $\tilde{v}, \tilde{f} \tilde{\mathcal{F}}_{i}$ is contained in a single octant. Since $n \leq 3$ and $\bigcup_{i} \tilde{f} \tilde{\mathcal{F}}_{i}$ is neighborhood of $\tilde{f}(\tilde{v})$, then either $\operatorname{deg}_{K}(v) \geq 6$ or $\bigcup_{i} \tilde{f} \tilde{\mathcal{F}}_{i}$ is contained in a single octant. However in the latter case $\operatorname{deg}_{K}(v)=3$, so the face disks $\mathcal{F}_{i}$ are not special, for a contradiciton.

Lemma 5.12 There is a fixed constant $c>0$ such that, if $f: S \rightarrow \mathbb{H}^{3} / \Gamma(P)$ is a cellular immersion with respect to a polyhedral structure $K$ on $S$, and if $f$ is in standard form, then $\operatorname{genus}(S) \geq c\left|K^{(2)}\right|$.

Proof Since $f$ is in standard form, the 2-skeleton of $K$ has a partition into special face disks. Let $K^{\prime}$ be the corresponding complex of $S$, and let $\mathcal{F} \in K^{\prime(2)}$.

Let $v \in \mathcal{V}(\mathcal{F}, 2)$, and suppose $\operatorname{deg}_{K^{\prime}}(v) \leq 3$. Since $\operatorname{deg}_{\mathcal{F}}(v)=2$, then we have $\operatorname{deg}_{K}(v)<2 * \operatorname{deg}_{K^{\prime}}(v) \leq 6$. Therefore by Lemma 5.11 $\operatorname{deg}_{K^{\prime}}(v) \geq 4$, for a contradiction. Therefore every $v \in \mathcal{V}(\mathcal{F}, 2)$ has $\operatorname{deg}_{K^{\prime}}(v) \geq 4$. Since $\mathcal{F}$ is special, $|\mathcal{V}(\mathcal{F}, 2)| \geq 5$, and so

$\left(^{*}\right)$ each $\mathcal{F}$ has at least five vertices $v$ with $\operatorname{deg}_{K^{\prime}}(v) \geq 4$. 
Let $V, E$ and $F$ represent the number of vertices, edges and faces in the complex $K^{\prime}$. For $\mathcal{F} \in K^{\prime(2)}$, let $V(\mathcal{F}, n)$ be the number of vertices which are distinct in $\mathcal{F}$ (not necessarily in $K^{\prime}$ ) and which have degree $n$ in $K^{\prime}$. We have:

$$
\begin{aligned}
\chi(S) & =V-E+F \\
& =V+(-1 / 2)\left(\sum_{v \in K^{\prime}(0)} \operatorname{deg}(v)\right)+F \\
& =-1 / 2\left(\sum_{v \in K^{\prime}(0)} \operatorname{deg}(v)-2\right)+F \\
& =-1 / 2\left(\sum_{\mathcal{F} \in K^{\prime}(2)} 1 \frac{V(\mathcal{F}, 3)}{3}+\sum_{\mathcal{F} \in K^{\prime}(2)} 2 \frac{V(\mathcal{F}, 4)}{4}+\sum_{\mathcal{F} \in K^{\prime}(2)} 3 \frac{V(\mathcal{F}, 5)}{5}+\ldots\right)+F \\
& \leq-1 / 2\left(\sum_{\mathcal{F} \in K^{\prime}(2)} \frac{1}{2}[V(\mathcal{F}, 4)+V(\mathcal{F}, 5)+\ldots]\right)+F \\
& \leq-1 / 2\left(\frac{1}{2} 5 F\right)+F, \text { by }(*) \\
& =-\frac{1}{4} F
\end{aligned}
$$

Since each face disk $\mathcal{F}$ can contain at most $\left|P^{(2)}\right|$ faces of $P$, then $\left|K^{(2)}\right| \leq$ $\left|P^{(2)}\right|\left|K^{\prime(2)}\right|$, and the lemma follows.

Combining Lemma 5.12 with Lemma [5.8, we get the following, which may be of some independent interest:

Proposition 5.13 For $P$ and $M$ as in Theorem 1.3 there is a constant $c$ such that every immersed incompressible genus $g$ surface in $M$ can be homotoped to a union of at most $c g$ faces of $P$.

We now resume the proof of the upper bound.

By Lemma 5.12, the total number of maximal face disks is linear in the genus, and so the number of edges on the boundary of these faces is linear in the genus also. The number of choices for the face-disks is then exponential in $g$, and the number of ways of gluing the face disks along the boundary edges is factorial in $g$, resulting in a factorial upper bound for the total number of immersions.

We now sketch the proof of the explicit bound given in Theorem 1.3. Let $K^{\prime}$ be a special face-disk complex for $S$. The above analysis gives $s_{2}(M, g) \leq$ 
$e^{c_{1} g}\left(\left|K^{\prime(1)}\right|\right)$ !, for some constant $c_{1}$. The proof of Lemma 5.12 shows that $\chi(S) \leq-\frac{1}{4}\left|K^{\prime}(2)\right|$, so $\left|K^{\prime(2)}\right| \leq 8 g-8$. Let $c(P)$ be the maximum number of edges in a face disk of $P$. Then $\left|K^{\prime(1)}\right| \leq c(P)\left|K^{\prime(2)}\right|$, and so $s_{2}(M, g) \leq$ $e^{c_{1} g}((8 g-8) c(P))$ !, and so for large $g, s_{2}(M, g) \leq e^{(8 c(P)+1) g \log (g)}$.

\section{$6 \quad$ Further Questions}

Theorem 1.2 prompts the question:

Question 1 Does the conclusion of Theorem 1.2 hold for every closed, hyperbolic 3-manifold?

Of course, even proving that $s(g, M)$ is not constantly zero on any $M$ is very difficult.

It is noteworthy that, in the examples of Theorem 1.2. the factorial growth was achieved entirely by quasi-Fuchsian surfaces. If Thurston's virtual fibering conjecture is true, there should always exist in addition a certain number of geometrically infinite immersions. However, the general constructions of $\pi_{1}$ injective immersions which are currently known seem to produce geometrically infinite examples only sporadically.

Question 2 Let $M$ be a closed hyperbolic 3-manifold, and let $i(g)$ denote the number of conjugacy classes of maximal, geometrically infinite surface groups of genus at most $g$ in $\pi_{1} M$. Does $i(g) / s(g) \rightarrow 0$ as $g \rightarrow \infty$ ? Does $i(g)$ have sub-factorial (or even polynomial) growth?

\section{References}

[1] B H Bowditch, G Mess, A 4-dimensional Kleinian group, Trans. Amer. Math. Soc. 344 (1994) 391-405 MathReview

[2] J Elstrodt, F Grunewald, J Mennicke, Groups acting on hyperbolic space, Springer Monographs in Mathematics, Springer-Verlag, Berlin (1998) MathReview

[3] D G James, C Maclachlan, Fuchsian subgroups of Bianchi groups, Trans. Amer. Math. Soc. 348 (1996) 1989-2002 MathReview

[4] Alexander Lubotzky, Counting finite index subgroups, from: "Groups '93 Galway/St. Andrews, Vol. 2", London Math. Soc. Lecture Note Ser. 212, Cambridge Univ. Press, Cambridge (1995) 368-404 MathReview 
[5] C Maclachlan, A W Reid, Parametrizing Fuchsian subgroups of the Bianchi groups, Canad. J. Math. 43 (1991) 158-181 MathReview

[6] Teruhiko Soma, Virtual fibers in hyperbolic 3-manifolds, Topology Appl. 41 (1991) 179-192 MathReview

[7] Peter Scott, Subgroups of surface groups are almost geometric, J. London Math. Soc. (2) 17 (1978) 555-565 MathReview

[8] William Thurston, The Geometry and Topology of Three-Manifolds, lecture notes, Princeton University (1978-1980) http://www.msri.org/publications/books/gt3m/

Mathematics Department, Rice University, Houston TX 77005, USA

Current address:

Mathematics Department, SUNY Buffalo, Buffalo NY 14260, USA

Email: mastersj@rice.edu, jdmaster@buffalo.edu

Received: 20 October 2004 